\title{
La « professionnalisation » des formations en géographie : spécificité disciplinaire?
}

Matthieu Pichon, Caroline Leininger-Frézal et Nicolas Douay

\section{(2) OpenEdition}

Journals

Édition électronique

URL : http://journals.openedition.org/cdg/1164

DOI : $10.4000 /$ cdg. 1164

ISSN : 2107-7266

Éditeur

UMR 245 - CESSMA

Référence électronique

Matthieu Pichon, Caroline Leininger-Frézal et Nicolas Douay, «La « professionnalisation » des

formations en géographie : spécificité disciplinaire? 》, Carnets de géographes [En ligne], 10 | 2017, mis en ligne le 30 septembre 2017, consulté le 19 avril 2019. URL : http://journals.openedition.org/ cdg/1164; DOI : 10.4000/cdg. 1164

Ce document a été généré automatiquement le 19 avril 2019.

\section{(†)

La revue Carnets de géographes est mise à disposition selon les termes de la Licence Creative Commons Attribution - Pas d'Utilisation Commerciale - Pas de Modification 4.0 International. 


\title{
La « professionnalisation » des formations en géographie : spécificité disciplinaire?
}

\author{
Matthieu Pichon, Caroline Leininger-Frézal et Nicolas Douay
}

1 La géographie telle qu'elle est enseignée à l'Université a connu de profondes mutations depuis les années 1960, parmi lesquelles figure la mise en place de cours et de diplômes destinés à sensibiliser ou développer des pratiques professionnelles chez les étudiants. Cette «professionnalisation " des enseignements touche des domaines variés: aménagement, urbanisme, mais également environnement, enseignement ou cartographie. Plus largement, ces mutations s'inscrivent dans un contexte institutionnel dans lequel le rapprochement des formations universitaires avec le monde du travail a été encouragé et valorisé par les autorités de tutelle (Déclaration de la Sorbonne, 1998; Déclaration de Bologne, 1999). Si la géographie universitaire - c'est-à-dire la discipline telle qu'elle est pensée, mise en œuvre et enseignée à l'université - a longtemps eu pour principal débouché l'enseignement, notamment secondaire (Rhein, 1982), ce n'est plus le cas aujourd'hui. A titre d'exemple, moins de $10 \%$ des cohortes de candidats admis au CAPES d'Histoire-Géographie sont géographes. La discipline s'est fortement engagée depuis les années 1960-1970 dans le développement de formations s'ouvrant ainsi à de nouveaux marchés d'emplois. Nous faisons l'hypothèse que cette mutation correspond à un double mouvement ascendant et descendant: descendant par effet de contexte des différentes injonctions institutionnelles (Ministère, Union Européenne, etc.) et ascendant par l'existence, au sein de la discipline, de questionnements et de débats épistémologiques à propos de son utilité en lien avec les stratégies d'investissement de « l'aménagement du territoire » par certains géographes.

2 Cette recherche de légitimité des acteurs les a poussés à repenser le contenu des formations proposées en géographie avec pour objectif la «professionnalisation » des cursus. Ces transformations, naturalisées par les acteurs, ont ainsi pu contribuer à une certaine redéfinition de la discipline et son identité. 
3 Pour explorer cette hypothèse, nous nous appuierons sur l'étude de la géographie en tant que discipline, dans ses relations avec des débouchés qualifiés de " professionnels » (hors de l'enseignement) et notamment avec l'aménagement en tant que processus d'action publique et marché d'emploi, dans la mesure où ce champ a souvent été considéré par les géographes comme un débouché ou un prolongement pour leur discipline ${ }^{1}$. Notre étude adopte par ailleurs une approche historique de cette dernière, qui invite à la contextualisation des enjeux contemporains de la géographie dans un temps plus long, compris entre les années 1970 et 2000. Nous proposons enfin une étude de cas plus récente, centrée sur l'Université Paris-Diderot: deux des co-auteurs y enseignent, et peuvent ainsi témoigner de leur expérience directe et pratique des formations qui y sont proposées. En outre, le choix de cette institution peut être justifié par l'affirmation (au moins dans l'ordre du discours), dès sa création au début des années 1970 et à la suite des événements de mai 1968, d'un projet d'interdisciplinarité et d'ouverture vers la société ${ }^{2}$ Ce choix découle aussi du positionnement du département de géographie qui se définit comme ayant une approche critique, innovante et un intérêt pour les questions de didactique $^{3}$ qui se traduisent, notamment, par le développement de formations professionnelles, par exemple en aménagement.

4 Pour valider notre hypothèse, nous nous appuierons sur une méthodologie quantitative et qualitative. Nous avons recours à plusieurs corpus issus d'un travail d'archives et de terrain. Afin d'offrir un panorama historique de l'ensemble des formations, nous avons dépouillé les Intergéo-Bulletin de 1968 à 1998. Publiés de façon trimestrielle depuis les années 1960 par le laboratoire INTERGEO puis le laboratoire PRODIG, les Bulletins se présentent comme des outils de liaison pour la communauté des géographes et fournissent toute une série d'informations sur des thématiques variées (recherches en cours, enseignements et diplômes, rapports, etc.). L'équipe éditoriale a notamment publié, en 1988 et 1998, deux guides des formations universitaires en géographie et dans des domaines proches, sur lesquels nous fondons une grande partie de nos analyses.

5 Pour notre étude de cas, un travail sur archives ${ }^{4}$ a été mené à la Bibliothèque Nationale de France et au service des archives de l'Université Paris-Diderot. Les archives n'étant pas toujours complètes, les documents trouvés ne permettent pas toujours d'établir la continuité dans l'évolution des formations. Nous avons recherché les maquettes et les plaquettes (documents de présentation à destination des étudiants) des formations proposées en géographie et en aménagement ainsi que les délibérations des instances universitaires (CEVU puis CFVU ; conseils d'UFR ; assemblés générales du département de géographie, etc.) des années 1970 à nos jours. Quatre entrées ont guidé cette recherche : l'aménagement, la "professionnalisation ", les stages en entreprise ou au sein d'organismes publics et la prise en compte du terrain dans les enseignements.

Trois autres corpus complètent enfin ces premiers :

7 - Deux séries d'entretiens semi-directifs ont été menées. Les premiers ont été réalisés auprès de sept enseignants-chercheurs de géographie avec pour objectif d'interroger leur identité professionnelle et plus particulièrement les liens faits entre la recherche et l'enseignement de la géographie. La professionnalisation est un des aspects abordés dans ces entretiens mais ce n'est pas le sujet central. Cette enquête a été menée dans le cadre du groupe sur l'enseignement supérieur du Laboratoire de didactique André Revuz sous la direction de Nicolas Grenier. Les seconds entretiens ont été conduits auprès de 10 enseignants-chercheurs de géographie et d'aménagement sur l'usage des cas et des exemples dans leurs enseignements, dans le cadre d'une recherche financée par l'Institut 
des Humanités de Paris en 2013. Ces deux enquêtes n'étaient pas destinées à explorer la question de la "professionnalisation " mais c'est un aspect qui ressort des entretiens notamment dans les pratiques pédagogiques. Les caractéristiques des enseignantchercheurs interrogés sont en annexe. Le lieu d'exercice des enseignants-chercheurs interrogés n'est pas précisé afin de garantir l'anonymat des collègues qui ont accepté d'être enquêtés. Dans l'ensemble du corpus, nous avons interrogé 10 hommes et 7 femmes parmi lesquels 14 sont maîtres de conférences et 3 professeurs. Un tiers des enseignantschercheurs ont une expérience dans le secondaire (6/17). La grande majorité (11/17) d'entre eux est impliquée dans la formation professionnelle, généralement dans le cadre de masters professionnels ou de la formation des enseignants.

- Une enquête par questionnaire a été menée en septembre 2015 auprès de l'ensemble des étudiants inscrits et présents en licence 1 de géographie et aménagement à l'Université Paris-Diderot. 88 étudiants ont répondu en respectant les conditions d'anonymat demandées. Cette enquête visait à identifier leur représentation de la géographie, ce qui a motivé leur choix d'étude et leur projet professionnel ${ }^{5}$.

L'ensemble de ces corpus nous permettra de montrer que la "professionnalisation" occupe une part croissante des formations proposées en géographie et en aménagement. En effet, l'insertion professionnelle est devenue une finalité explicite de ces formations.

La « professionnalisation » de ces dernières s'inscrit dans un contexte politique favorable à cette évolution, ainsi que dans un contexte de crise économique. Elle a aussi à voir avec le renouvellement de la discipline : les géographes ont engagé une réflexion autonome et parallèle sur la "professionnalisation ", tant et si bien que celle-ci semble être devenue une composante de l'identité disciplinaire en géographie et en aménagement. Cela a un fort impact sur les pratiques pédagogiques qui intègrent des pratiques professionnelles : cela se traduit de différentes manières, et notamment par des ateliers qui répondent à une commande fictive ou réelle d'un acteur public ou privé en mettant en scène directement la pratique ou bien par des sorties de terrain au plus près des acteurs professionnels.

\section{Entre injonctions institutionnelles et réflexions internes : géographie et « professionnalisation »}

11 La "professionnalisation» des formations de géographie s'inscrit dans le cadre des réformes de l'enseignement supérieur.

\section{L'injonction à la « professionnalisation »}

12 Du point de vue des politiques publiques, la " professionnalisation » est présentée comme un levier de réforme de l'Université. "Parmi d'autres objectifs de la refonte des formations supérieures, la "professionnalisation » apparait comme une notion-clé au sein des textes officiels européens et nationaux» (Stavrou, 2011 : 94). L'accent mis sur la «professionnalisation » découle d'une combinaison de facteurs multiples opérant sur une période d'une quarantaine d'années.

13 A l'échelle nationale, la massification de la population estudiantine a joué un rôle clé. Il s'agissait d'adapter l'université à l'hétérogénéité croissante de son public. Les étudiants issus de milieux populaires ont des attentes différentes que ceux issus de catégories socio- 
professionnelles favorisées, en termes d'utilité des apprentissages réalisés notamment sur un plan professionnel (Paivandi, 2011). La "professionnalisation » vise aussi à répondre aux besoins du marché du travail (Vincens et Chirache, 1992) dans une logique qualifiée d'adéquationniste par Jean-François Giret et Stéphanie Moulet (2008:4): «il s'agit de répondre à des besoins précis du marché du travail en termes de niveaux et de spécialité ». C'est dans cette perspective que se développent les premiers cursus dit "professionnalisants» dans les années 1960 et 1970 avec la création de nouveaux diplômes ou structures universitaires d'abord dans le premier cycle ${ }^{6}$ puis dans le second ${ }^{7}$. La création de filières technologiques et professionnelles continue dans les années 1980 et 19908. "Pourtant, jusqu'au milieu des années 1990, et bien qu'encouragée par le ministère, la mobilisation collective autour de la "professionnalisation » est faible » (Gayraud, Simon-Zarca et Soldano, $2011: 10$ ).

14 L'émergence d'une volonté politique à l'échelle européenne de réformer l'enseignement supérieur a joué un rôle moteur de changement depuis les années 2000 (Agulhon et Convert, 2011: 6). Le point de départ semble être la prise de conscience par les leaders européens de la perte de compétitivité des pays européens à l'échelle mondiale (Gayraud, Simon-Zarca et Soldano, 2011), ce qui a conduit à questionner l'efficacité des formations supérieures. Il s'agit d'améliorer la qualité des formations proposées pour accompagner les pays européens dans leur entrée dans une «économie de la connaissance » et accroître leur productivité (Agulhon et Convert, 2011).

Si la France a au départ une position distanciée par rapport à ces réformes, le pays entre dans les logiques européennes dans les années 2000 avec la mise en place du système LMD, des ECTS (Gayraud, Simon-Zarca et Soldano, 2011), des licences professionnelles (2000) et des masters professionnels (2002). La loi sur les Libertés et Responsabilités des Universités $\left(\mathrm{LRU}^{9}\right)$ assigne aux universités une mission d'orientation et d'insertion professionnelle. Le Plan pluriannuel de réussite en licence mis en place la même année va dans le même sens : il "propose un cadre pour rénover le contenu du diplôme afin de l'inscrire dans une logique de maîtrise progressive des connaissances et des compétences » (Gayraud, Simon-Zarca et Soldano, 2011: 20). Cela s'est traduit par la mise en place de modules obligeant l'étudiant à travailler son projet personnel, de stages en milieu professionnel au cours de la licence et d'un référentiel de compétences en licence.

Parallèlement, la formation destinée aux enseignants se veut plus professionnalisante avec notamment la mise en place des Instituts universitaires de formation des maitres (IUFM en 1989). En 1991, une "épreuve professionnelle " est créée à l'oral du CAPES d'histoire-géographie bien qu'aménagée en 1993 (Bourdon et al., 2015 ; Delacroix, 2015). La deuxième année de formation des IUFM y est dédiée avec la mise en place d'un "mémoire professionnel» dans lequel les futurs enseignants doivent acquérir une posture réflexive sur leurs pratiques de classe. La finalité professionnalisante de la formation des enseignants est réaffirmée en 2012. " «Professionnalisation » devient le mot valise concernant le recrutement des enseignants » (Bourdon et al., 2015, p. 166). Les épreuves sur dossier à l'écrit et à l'oral du CAPES comportent une dimension dite "didactique ", présentée comme en prise avec les pratiques professionnelles enseignantes. Alors que la place de la géographie est réaffirmée dans les concours de l'enseignement avec une réelle bivalence histoire et géographie, les géographes se sont peu exprimés sur la formation des enseignants contrairement aux historiens ${ }^{10}$. C'est en partie ce qui justifie que nous ayons décidé de ne pas traiter la "professionnalisation " des formations enseignantes dans cet article. 
Les politiques tant françaises qu'européennes qui ont impulsé la « professionnalisation » des formations universitaires, reposent sur une conception marchande et libérale de l'université et de ses formations. Cela justifie leur mise en concurrence dans le but d'améliorer la productivité et la performance des pays concernés (Agulhon et Convert, 2011). Mais alors que la «professionnalisation » devient un mot d'ordre des politiques d'enseignement supérieur, la notion renvoie à des réalités multiples.

Le terme de "professionnalisation» est polysémique (Stavrou, 2011 ; Agulhon et Convert, 2011 ; Wittorski, 2008 ; Vincens et Chirache, 1996 ; Rose, 2008). La " professionnalisation» renvoie à :

Des diplômes construits autour d'une profession ou d'un champ professionnel comme les facultés de médecine, les écoles d'infirmièr.e.s, écoles d'architecture etc. «Dans un premier cas, la notion de «professionnalisation » exprime un choix d'orientation globale, une finalité explicitement reliée à l'emploi : on dira alors qu'une filière est professionnelle si elle débouche en principe directement sur un emploi » (Rose, 2008 : 52). Les formations en urbanisme et en aménagement ou en enseignement (master MEEF) relèvent de cette acception du terme : professionnalisation signifie spécialisation. qu'elle exige la confrontation à la pratique et l'apprentissage de savoirs opérationnels, qu'elle recouvre la maîtrise de savoirs pratiques, l'apprentissage des conditions concrètes du travail en entreprises, l'acquisition de capacités professionnelles transversales, etc. » ( op. cit.). Les stages, les modules de projet personnel de l'étudiant, les ateliers mis en place dans les formations de géographie ou d'aménagement ou encore les stages de terrain peuvent répondre à cette définition.

21 La participation de "professionnels » à l'équipe pédagogique : «Enfin la "professionnalisation» manifeste une conception du métier de formateur, une filière professionnelle faisant intervenir des « professionnels » et exigeant des partenariats avec les entreprises. » (op. cit.). A noter que dans cette perspective les enseignants-chercheurs ne sont pas considérés comme des professionnels!

Selon Stavrou (2011, §23), la valeur des formations qualifiées de professionnalisantes dépend de «la nécessité socio-économique de la formation », du "taux d'insertion professionnelle des diplômés et [des] débouchés visés » et des « partenariats établis ». Ces critères figurent parmi ceux déployés par les institutions de tutelle et par l'AERES pour évaluer ces formations. L'injonction institutionnelle à la " professionnalisation » de ces dernières a profondément modifié leurs contenus et la manière de les concevoir dans l'enseignement supérieur (Stavrou, 2011; Agulhon, 2007). Les contenus d'enseignement ne sont pas seulement définis à partir d'une discipline, de son évolution et de ses enjeux mais aussi à partir de pratiques professionnelles et d'autres enjeux de nature sociale. Les compétences considérées comme utiles à acquérir pour exercer un métier servent d'étalon pour définir les contenus de formations. Cela s'inscrit aussi dans un contexte de substitution de la «qualification" - stable et négociée collectivement - par les " compétences » individuelles et en constante évolution. C'est une profonde modification $\mathrm{du}$ processus de conception des formations, qui incombaient jusque-là uniquement aux enseignants-chercheurs.

La géographie française a connu et connaît les trois formes de "professionnalisation » déclinées ci-dessus. A la lecture de notre article, il faudra donc garder en tête cette polysémie lorsque nous utilisons ce terme, même s'il apparaîtra que nous insistons plus 
sur l'une ou l'autre des acceptions selon les moments ${ }^{11}$. Dans la géographie française, les références à la "professionnalisation » (très régulièrement exprimées sous la forme du syntagme de "géographie professionnelle») ont souvent désignées une volonté d'insertion dans des marchés du travail hors de l'enseignement et de la recherche. Cette professionnalisation de la géographie, si elle s'inscrit et répond aux différentes réformes et injonctions, s'explique aussi en partie par des facteurs plus endogènes à la discipline : elle participe selon nous de la crise et des transformations que connait la discipline en France à partir des années 1970, dans le même temps qu'elle en découle, au moins en partie.

\section{La « professionnalisation » entre les années 1970 et 1990 : un enjeu de survie pour la géographie?}

La géographie française a connu au tournant des années 1970 un «malaise » suivie d'une "crise $»^{12}$ (Orain, 2009, 2015) qui ont constitué le point de départ d'une série de transformations diverses (épistémologiques, méthodologiques, etc.), particulièrement prégnantes dans les années 1970-1980. Ces renouvellements ont aussi concerné les formations universitaires en géographie, tant du point de vue de leur organisation (maquettes, modalités d'obtention du diplôme, etc.) que de leurs débouchés. L'une des caractéristiques de la géographie française, comme discipline savante et universitaire, est d'avoir entretenu des liens étroits avec la géographie scolaire (Rhein, 1982) et l'enseignement (notamment secondaire), ce dernier ayant été au moins jusqu'aux années 1970-1980 le «premier marché » et le « débouché naturel » (Robic, 2006) de la discipline. Néanmoins, on observe à partir des années 1970 une ouverture progressive à d'autres formes de "professionnalisation ", dans des domaines variés : aménagement, urbanisme, environnement, administrations territoriales... Cette ouverture constitue à la fois une réponse à la réduction des postes offerts au sein de l'Education nationale ${ }^{13}$, ainsi qu'une adaptation face aux souhaits d'orientation parfois exprimés par les étudiants et qui semblent se diversifier. Mais elle relève aussi d'une préoccupation, déjà présente dans les années 1960, quant au rôle et à la place de la géographie face à des disciplines concurrentes, notamment dans leurs rapports aux mondes économique, politique et administratif - face à la "demande sociale ", pour le dire plus brièvement - dans une période de décentralisation et de montée en puissance des pouvoirs locaux qui offrent de nouvelles opportunités d'emploi.

Ces préoccupations s'expriment au sein de différentes scènes collectives de la discipline (journées, revues, rapports, etc.). Sans l'épuiser complètement, nous pouvons ici retracer l'expression de ces inquiétudes ainsi que son évolution.

Dès 1968 et suite aux "événements ", ces questions sont posées par les "Etats généraux de la géographie » organisés à Tours en juillet (Collectif, 1968). Réunissant des délégations enseignantes et étudiantes des instituts de géographie, ces journées sont l'occasion de réflexions thématiques organisées en différentes commissions. L'une d'entre elles s'intéresse notamment aux "débouchés » de la géographie, qu'elle classe en deux types: les débouchés dans l'enseignement et ceux « hors de l'enseignement ».

Cette dernière question s'accompagne de propositions, comme la mise sur pied «[ $d$ '] Instituts universitaires de Formation Professionnelle ou Ecoles d'Application orientant les géographes [...] vers des domaines précis de l'activité économique (aménagement rural, collectivités locales, Instituts d'Urbanistes, etc.) », liée au «danger de voir la place de la 
Géographie trop mesurée dans les équipes pluridisciplinaires d'aménagement et [...] dans de nombreux secteurs de l'Activité économique ». Cela suscite néanmoins des réactions de doute: "quelles personnes désire-t-on former?», demande ainsi Pierre Clergeot, représentant de la Sorbonne; s'agit-il «d'adapter les gens aux débouchés actuels ou [de] s'orienter différemment?». Des critiques plus vives sont exprimées, comme celle de Maurice Allefresde (Lyon) qui souhaite " éviter de souscrire à la demande, car on risque de ne former que des manœuvres", ou bien encore celle de René Rochette, délégué grenoblois : il « ne faut plus former des travailleurs sous-payés pour servir le capitalisme d'Etat ou le capitalisme privé ».

Définis par contraste avec ce qui constitue encore l'orientation majeure des formations en géographie, les débouchés "hors de l'enseignement» suscitent ainsi des positions hétérogènes quant à la question du rapport des géographes avec les emplois situés hors de l'école et de l'université. Si certains craignent que la non-implication de la discipline dans les domaines de « l'activité économique » se signe par sa relégation dans le concert des sciences humaines et sociales, d'autres en revanche manifestent une certaine défiance quant à cette implication - ce qui, d'une certaine manière, peut rappeler les divergences exprimées dans les débats entre " géographie active » et « géographie appliquée » dans la décennie écoulée. Il apparaît par ailleurs, au terme de ces journées, que l'insertion professionnelle des diplômés en géographie reste relativement mal connue. C'est ainsi que l'Assemblée générale adopte notamment une proposition visant à demander l'organisation «au plan national d'un colloque chargé d'étudier les débouchés hors de l'enseignement ", appuyé par une "enquête " préalable portant notamment sur la "situation » des anciens étudiants en géographie, sur les « perspectives » et la « situation matérielle et sociale des géographes » hors de l'enseignement, ainsi que sur les « lacunes dans la formation universitaire géographique pour l'emploi hors de l'enseignement ».

Ces "états généraux » sont assez révélateurs d'une série de motifs qui reviennent s'exprimer régulièrement dans la géographie française à partir des années 1970 . Ces motifs mêlent inquiétudes, conscience des opportunités nouvelles qui s'ouvrent avec l'émergence de nouveaux marchés d'emplois, et volontés d'élargir l'éventail des débouchés offerts aux étudiants. A l'image des "états généraux ", ces préoccupations se manifestent souvent lors de débats, réunions ou commissions diverses.

Ainsi, par exemple, lors des « Journées géographiques » de Rennes ${ }^{14}$, en 1972, les membres de la commission «Enseignement et méthodologie » soulignent le "rétrécissement des débouchés dans l'enseignement » et expriment leurs craintes :

«[...] si l'on place 1300 professeurs par an actuellement (12000 candidats aux concours), on tendrait à terme vers le simple renouvellement dû aux départs à la retraite, soit vers $200 .$. Une réflexion sur les débouchés est devenue vitale » (CNFG, $1972: 202)$.

31 Dix années après, on retrouve des réflexions analogues dans les brochures des cursus proposés en géographie à l'université Paris Diderot :

«La géographie débouche sur les carrières d'enseignement (voir ci-dessus). Mais elle prépare également aux carrières d'aménagement et de recherche. Dans les deux cas cependant, et comme dans beaucoup d'autres disciplines, les débouchés sont restreints. Les moyens affectés à la formation des enseignants ou des professionnels sont très réduits, ce qui gène considérablement le développement de ces orientations. Notre U.E.R. subit comme partout la sévère crise de l'université et de l'emploi » (Université Paris 7, plaquette de présentation du Cursus de géographie, 1981-1982) 

En organisée une restitution des réflexions de groupes de travail sur le thème de " la géographie, son contenu et sa place dans la société d'aujourd'hui face aux problèmes actuels " ${ }^{15}$. L'un des groupes travaille sur la question de «la géographie pour les géographes professionnels ». Si l'on tente de synthétiser les conclusions de ce groupe, on doit d'abord considérer que les "géographes professionnels» constituent un contingent encore assez réduit (environ $10 \%$ des diplômés du moment, selon le croisement de plusieurs enquêtes). Pour la plupart et hormis des cas exceptionnels, leur situation est précaire et relativement déconnectée des enseignements qu'ils ont reçus: une majorité vit de contrats de vacation et exerce en dehors de sa spécialité de départ. Quant aux employeurs, ils montrent une certaine insatisfaction face au manque de «connaissances économiques, de techniques d'enquêtes, [de] maniement des statistiques, [...] d'expérience pratique » de ces diplômés.

34 Les discussions qui ont lieu suite à la restitution du groupe de travail sont intéressantes : tout d'abord, les participants identifient deux groupes parmi les géographes professionnels, dont l'un est "plus résolument tourné vers l'aménagement et l'urbanisme». Relativement à ce marché d'emploi, des recommandations sont donc faites: renforcer l'enseignement de compétences techniques (statistiques, traitements graphiques, " notions juridiques sur l'aménagement et l'urbanisme, informatique... ") et ce notamment pour ceux qui se dirigent vers l'aménagement-urbanisme (qui devraient aussi avoir des " éléments de comptabilité, de gestion et de méthodes de financement »). On préconise aussi une refonte partielle du DEUG, en réduisant la part de l'histoire (qui oriente plus vers les concours de l'enseignement) pour enseigner plutôt des «UV supplémentaires » sur les domaines en question. Pour favoriser l'expérience pratique des diplômés, il est aussi proposé d'intégrer des stages au sein des cursus. Enfin, la question de l' "ouverture pluridisciplinaire» est posée, car «le géographe doit avoir l'habitude de travailler avec des équipes et être capable d'en comprendre les autres représentants ».

De fait, des transformations des enseignements ont bien lieu dès les années 1970 : l'enquête menée en 1981 par le CNFG (CNFG, 1983) et rapportée dans Intergéo-Bulletin souligne par exemple que la "raréfaction des postes à l'agrégation et au CAPES dans les 10 dernières années a amené beaucoup d'UER [...] à modifier le contenu de la licence dite d'enseignement, à introduire des enseignements nouveaux afin de faciliter l'insertion de leurs diplômés dans le secteur privé ou semi-public » (p. 98).

36 Ces préoccupations s'expriment aussi chez les étudiants eux-mêmes, qui affirment, comme lors de journées de travail à Strasbourg en $1986^{16}$, que « la géographie est plus qu’une discipline universitaire refermée sur elle-même, les géographes ont d'autres compétences que celles de l'enseignement, mais celles-ci sont peu connues [et qu'] il existe [dans cette période] une demande potentielle pour [leurs] formations». Il faudrait alors, disent-ils, "proposer nos compétences et [...] nous insérer pleinement dans les opérations d'aménagement et de gestion des milieux urbains, ruraux et naturels » (Collectif, $1986: 3$ ).

37 On pourrait multiplier à l'envie les citations et les exemples qui parsèment les années 1970-1980 pour la géographie française ${ }^{17}$ : ces considérations sur les formations, notamment en aménagement et en urbanisme, sont très récurrentes et préoccupent les géographes au moins jusqu'aux années 1990. 
se traduit enfin par un foisonnement d'enquêtes et de rapports sur la question des débouchés. Sans tous les citer, on peut penser néanmoins, pour le début des années 1970, à l'« Enquête sur les applications de la géographie et les débouchés pour les étudiants » réalisée par Michel Phlipponneau en 1971 (Phlipponneau, 1972: 88), dans laquelle il considère que "le passage [...] à une [...] étape marquée par l'entrée massive de jeunes géographes dans des carrières nouvelles ne sera pas aisé ». Ou bien encore à l'année 1981-1982, durant laquelle est réalisée une enquête sur "l'enseignement de la géographie dans les universités françaises » (CNFG, 1983) que viendra compléter celle de l'AFDG en 1987 auprès des étudiants qui étaient inscrits en maîtrise de géographie et d'aménagement en 1981-1982. Les rédactrices du rapport, Nicole Girard et Françoise Plet, y considèrent que si «Géographie et Aménagement sont pourtant des qualifications qui intéressent et répondent à des besoins importants ", les " débouchés se font plus rares aujourd'hui " pour les géographes dans les domaines de l'« analyse et la gestion de l'espace» (AFDG, $1987: 86-87$ ). Le rapport se conclut ainsi sur l'idée que les

« diplômes de Géographie, même ceux d'Aménagement [...] souffrent aujourd'hui de n'avoir pas vraiment réussi à s'imposer comme "sésames" professionnels durant les périodes de plein emploi » (ibid., p. 89). Ainsi, il est toujours nécessaire d'adopter une « démarche active de valorisation de la discipline hors de son contexte scolaire et universitaire» (ibid., p. 90).

Cette "valorisation» est d'ailleurs déjà entamée par une série de groupes qui se constituent dès les années 1970 pour promouvoir les débouchés «professionnels » de la géographie: Association professionnelle des géographes (1972), association Géographie active (1979) qui s'intéresse notamment à «l'aménagement du territoire», ou encore association Géopratiques évoquée précédemment. Ces associations produisent elles aussi des enquêtes recensant les géographes exerçant ailleurs que dans l'enseignement.

La géographie universitaire connait, entre les années 1970 et 2000, une diversification et une multiplication de son offre de formation, appuyées sur les réformes successives des diplômes à l'échelle nationale. La "professionnalisation » des cursus de géographie participe de ces transformations: plutôt marginale et informelle au début des années 1970, l'insertion des diplômés dans d'autres marchés que celui de l'enseignement devient petit-à-petit majoritaire, mais aussi plus organisée et structurée. D'une situation initiale où l'on ne trouve que quelques étudiants qui parviennent à trouver un emploi par le biais d'interconnaissances, on passe finalement à un paysage universitaire où une bonne partie des formations s'organisent autour de l'insertion professionnelle de leurs diplômés dans des domaines variés, souvent facilitée par la réalisation de stages longs et par l'existence d'associations faisant le lien entre université et marché du travail. L'ouverture des formations de la géographie à la "professionnalisation » s'appuie par ailleurs sur les transformations de l'action publique, notamment à partir des années 1980: la décentralisation, tout comme les évolutions de l'aménagement du territoire, tendent à mettre en avant le "local » et son " développement ", qui vise à aménager et développer les territoires par le bas grâce à la mobilisation des acteurs de la société civile.

41 Les rapports réalisés tout au long de cette période illustrent bien ces évolutions. Au début des années 1970, la «professionnalisation »- hors enseignement - semble encore anecdotique et informelle : comme le souligne M. Phlipponneau après l'enquête de 1971, ce sont « presque toujours [...] les contacts personnels entre l'universitaire [...] et [l'employeur] qui permettent au premier de suggérer qu'un jeune géographe pourrait assurer un emploi créé par le second» (Phlipponneau, 1972: 88). Le nombre d'étudiants recrutés varie beaucoup selon 
les instituts et selon les années, mais reste malgré tout assez confidentiel puisque «le nombre annuel d'emplois offerts doit être compris entre 60 et 80 ", ce qui est "très limité par rapport aux étudiants qui terminent leurs études de géographie par la préparation des concours » (p. 93). Aussi y a-t-il pour M. Phlipponneau inadaptation de "la formation des étudiants » avec des «carrières nouvelles»: les cursus de géographie devraient donner des "éléments d'économie, de droit administratif, de comptabilité publique " plutôt que de "l'histoire ancienne et du Moyen-Âge» (p. 93). Pour ce qui est des domaines effectivement investis par ces rares - géographes "hors de l'enseignement», il s'agit essentiellement de " l'administration " (notamment les ministères de l'Environnement et de l'Agriculture), les " organismes régionaux de développement économique, les bureaux d'études privés s'intéressant à l'urbanisme et à l'aménagement régional » (p. 93).

Ce sont sensiblement les mêmes débouchés que l'on retrouve dans les résultats de l'enquête du CNFG menée en 1981 (CNFG, 1983). Les informations sont certes partielles elles portent seulement sur des DEA des universités d'Aix, Toulouse, Nantes, Tours et Nice - mais permettent d'apprécier une certaine stabilité : entre les conseils régionaux, les DDE et DDA, agences d'urbanisme, bureaux d'études, collectivités locales ou bien encore les ministères, c'est tout un monde de l'administration locale, centrale ou déconcentrée généralement publique - des "études ", de l'aménagement et de l'urbanisme qui semble devenir le principal pourvoyeur d'emplois pour les géographes " professionnels ».

Les informations recueillies par l'enquête de l'AFDG réalisée en $1987^{18}$, plus détaillées, ne sont pas très différentes (AFDG, 1987). L'enseignement représente toujours le débouché le plus important mais n'est plus majoritaire (un tiers des répondants sont enseignants, un peu moins de la moitié sont employés par des établissements d'enseignement). En revanche, les "bureaux d'études", les "sociétés d'aménagement diverses, publiques, parapubliques ou privées » et les collectivités locales sont les «trois catégories d'employeurs qui offrent les emplois les plus proches des formations en Géographie et Aménagement » (p. 77), et représentent environ $18,5 \%$ des répondants. L'horizon professionnel des géographes se trouve ainsi dans le secteur public, même si un bon tiers des répondants travaillent dans le privé - mais pas toujours en lien avec leur formation de géographie ou d'aménagement. Si l'on s'intéresse au type d'emploi occupé, le second groupe le plus important après les enseignants est celui des chargés d'études (dans le public ou le privé) - environ un quart des enquêtés - qui exercent dans les domaines de l'urbanisme, des études économiques, du développement touristique ou local, de l'aménagement rural, ou bien encore dans la cartographie, l'informatique ou la télédétection (p. 78). Vient ensuite le groupe des " cadres administratifs et d'entreprise ». Ces deux groupes représentent au total « un effectif de réponses légèrement plus important que celui des enseignants", et se distinguent par une " qualification élevée " - il s'agit généralement de titulaires d'un DEA ou d'un DESS, ce dernier cas étant d'ailleurs en développement (un quart des enquêtés) alors qu'il était " encore relativement rare [quelques années auparavant] en géographie et aménagement » (p. 76) et par une "formation diversifiée» (leur cursus a pu se poursuivre en dehors de la géographie, celle-ci représentant alors une forme de préalable vers d'autres disciplines ou cursus). Notons enfin qu'un peu moins de la moitié des répondants considèrent que la formation qu'ils ont reçue n'est pas en adéquation ou en rapport avec la formation qu'ils ont reçue. Si l'autre moitié considère qu'il y a bien adéquation, ce sont surtout les enseignants qui répondent positivement et, dans une moindre mesure, les chargés d'études (pp. 87-88). 
$44 \mathrm{Au} \mathrm{vu} \mathrm{de} \mathrm{ces} \mathrm{différents} \mathrm{éléments,} \mathrm{il} \mathrm{semble} \mathrm{que} \mathrm{l'orientation} \mathrm{et} \mathrm{l'insertion}$ professionnelles des étudiants géographes ait connu quelques évolutions. La plus marquante est évidemment le passage d'une géographie formant avant tout à l'enseignement à une géographie dont les débouchés se diversifient. Cela n'enlève en rien l'importance de l'enseignement et des approches plus «théoriques » de la géographie comme issue privilégiée des études de géographie, mais la part qu'ils occupent est largement réduite par rapport aux années 1970. L'autre évolution majeure, c'est la structuration accrue de la spécialisation vers des professions "hors enseignement ", notamment par le biais de la création des DESS en $3^{\mathrm{e}}$ cycle (mais aussi de diplômes de cycles inférieurs comme les MST). Les géographes ont aussi su capter pour partie l'enseignement de "l'aménagement", en tentant parfois de naturaliser le lien entre celui-ci et la "géographie » (à ce titre, de nombreuses formations en " géographie » se sont vues requalifiées pour devenir des formations " en géographie et aménagement »), ce qui ne fut pas sans susciter quelques critiques (voir par exemple Merlin, 1991). Mais ce qui marque surtout, c'est la relative inertie des types de débouchés vers lesquels les diplômés de géographie se dirigent. On retrouve sur toute la période les mêmes domaines d'action (aménagement, urbanisme, action publique locale...) et les mêmes «lieux " d'activité (le monde des "études", public ou privé, celui des collectivités locales et territoriales...). En dehors de l'enseignement, il semble ainsi exister un champ assez stable de débouchés habituels pour les géographes - sans nécessairement que ceux-ci constituent leur chasse gardée, puisque d'autres disciplines peuvent se disputer ces emplois.

\section{Un tournant dans les années 1990 ?}

Qu'en est-il alors à la fin des années 1990 ? A partir de 1988, le laboratoire INTERGEO ${ }^{19}$ qui publie les Bulletins éponymes commence à faire paraître des répertoires de formations destinées aux géographes. Présentant les diplômes proposés dans les différentes villes de France, assortis de leurs débouchés et de l'organisation des études, ces répertoires découlent très nettement $\mathrm{du}$ contexte de préoccupation croissante sur l'insertion professionnelle des étudiants-géographes.

Ainsi, l'introduction du premier répertoire, paru en 1988, souligne-t-elle le «caractère lacunaire des informations disponibles sur le contenu des formations existantes [et] leurs débouchés " (AFDG \& INTERGEO, 1988: 3) malgré la multiplication des rapports et débats s'intéressant aux emplois occupés par les géographes. D'une certaine façon, ces répertoires s'inscrivent eux aussi dans cette série d'enquêtes sur d'anciennes cohortes d'étudiants: celui de 1988 par exemple est réalisé à partir d'un questionnaire dont l'initiative revient à l'AFDG et à INTERGEO. Il permet alors d'établir une liste des diplômes qui «porte[nt] le label Géographie» mais aussi - il faut le noter - «toutes les formations traitant de l'espace géographique et des territoires, au sein desquelles les compétences des géographes ont leur place» (p. 4, ibid.). Il n'est donc pas étonnant de retrouver dans ces répertoires des formations appartenant à des unités ou départements de droit, de gestion, de tourisme, d'environnement, etc. Si le choix d'inscrire telle ou telle formation aux répertoires revient bien entendu à leurs auteurs et à leur points de vue propres, il n'en demeure pas moins que ces catalogues donnent des indications sur la représentation de la discipline et de ses débouchés à la fin des années 1980 et 1990 (le répertoire suivant date de 1998, voir PRODIG, Briend, \& Vélard, 1998). Ils informent implicitement sur l'horizon 
professionnel attendu des géographes, sur les débouchés pour lesquels ils se considèrent légitimes. Autrement dit, et même si nous utilisons ces répertoires surtout comme des sources ici, ils n'en constituent pas moins une forme de matérialisation de la préoccupation professionnelle de la géographie française dans les années 1980-1990, et prennent la suite des nombreux débats qu'a connus la discipline à propos de cette question.

Pour essayer de donner à voir les évolutions quant à la "professionnalisation » des géographes, nous pouvons nous appuyer sur les informations recueillies par INTERGEO. Sans prétendre à l'exhaustivité, nous voudrions néanmoins dégager quelques lignes directrices de ces évolutions. Les répertoires font l'inventaire de différents types de diplômes, de la licence au DEA, en passant par les MST et les DESS. Nous voudrions partir de ce dernier cas, à la fois parce qu'il s'agit de diplômes dont les débouchés se trouvent « hors de l'enseignement » et de l'Université, mais aussi parce qu'ils constituent - en tant que diplômes de 3e cycle - une étape plus avancée dans la spécialisation professionnelle des étudiants.

Or, le nombre de DESS a connu une progression importante dans la décennie 1990 (voir figure 1). Ainsi, en 1988, toutes disciplines confondues, INTERGEO dénombre 42 DESS auxquels peuvent prétendre les étudiants-géographes; en 1998, cette offre a plus que doublé (+107\%) puisque 87 DESS sont répertoriés. Dans cet ensemble, si l'on se concentre uniquement sur les DESS qui sont dispensés dans le cadre de composantes de géographie (facultés, départements, UER de géographie ou mêlant la géographie et d'autres disciplines) ou qui touchent à des domaines assez proches ${ }^{20}$, il en reste 48 . Enfin, si l'on adopte un regard plus strict encore, 37 DESS sont des formations relevant de composantes strictement géographiques - cela exclut notamment la plupart des formations en urbanisme, qui relèvent d'instituts autonomes. A titre de comparaison, le nombre de DEA présentés dans le répertoire Intergéo passe de 91 en 1988 à 61 en 1998 (toutes composantes confondues dans les deux cas), ce qui représente une baisse d'un tiers environ (-33\%). Ces différences peuvent tenir aussi bien à des transformations réelles qu'à des évolutions dans le panel considéré par l'équipe d'Intergéo et il ne faut donc pas s'aventurer à en tirer des conclusions définitives. 
Figure 1. Evolution de l'offre de DESS de géographie entre 1974 et 1996

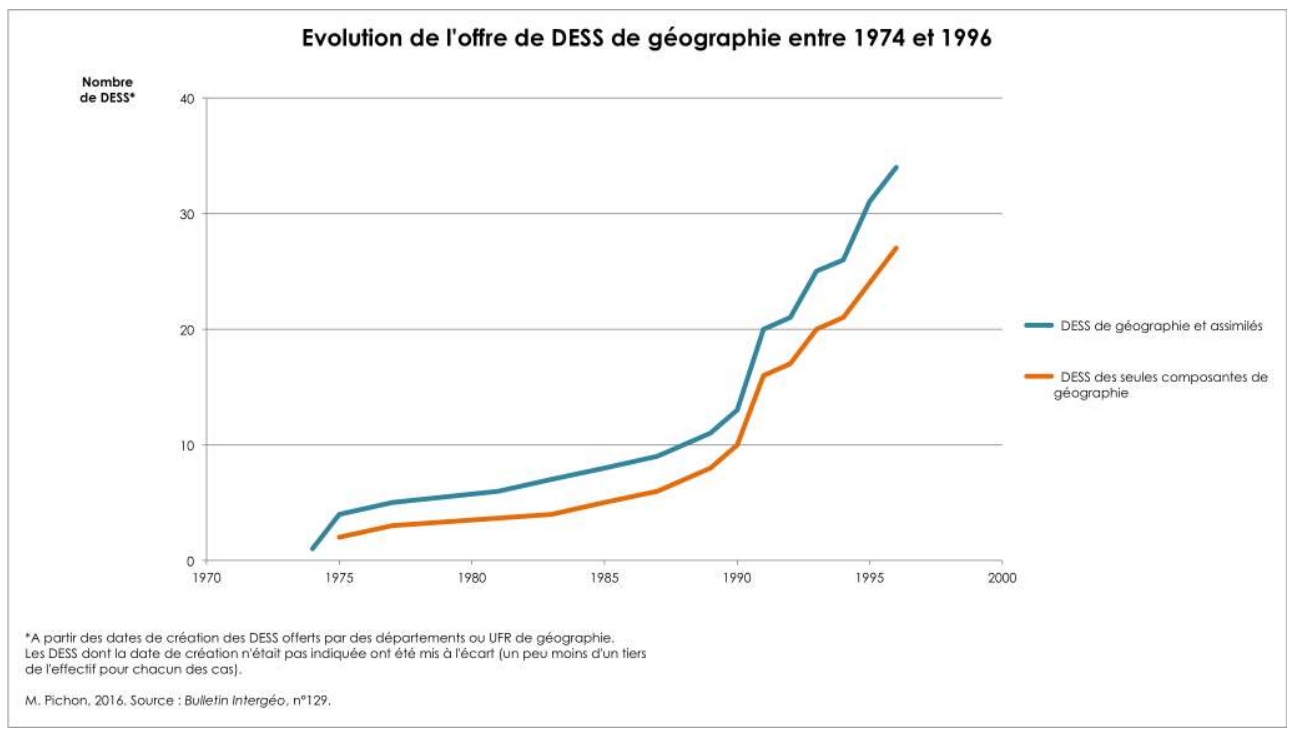

Crédits : Pichon, 2016

Dans le prolongement des données fournies par les enquêtes successives des années 1970-1990, nous avons voulu déterminer quels étaient les débouchés principaux visés par les DESS présentés par Intergéo en 1998, à partir de trois informations : leurs intitulés, les débouchés visés indiqués par chacune des fiches de diplôme, et enfin les débouchés recodés par nos soins pour obtenir des catégories un peu plus générales ${ }^{21}$. Intitulés des DESS

En considérant l'ensemble des 87 DESS, les domaines qui ressortent le plus sont ceux de l'aménagement, du développement (généralement du développement local, économique ou touristique), de la gestion (de l'espace, des aménagements touristiques, etc.) et, dans une moindre mesure, de l'urbanisme et de l'environnement.

Figure 2 : Termes les plus représentés dans les intitulés de DESS (1998)

Termes* les plus représentés dans les intitulés de DESS (1998) (Corpus : ensemble des DESS présentés par Intergéo)

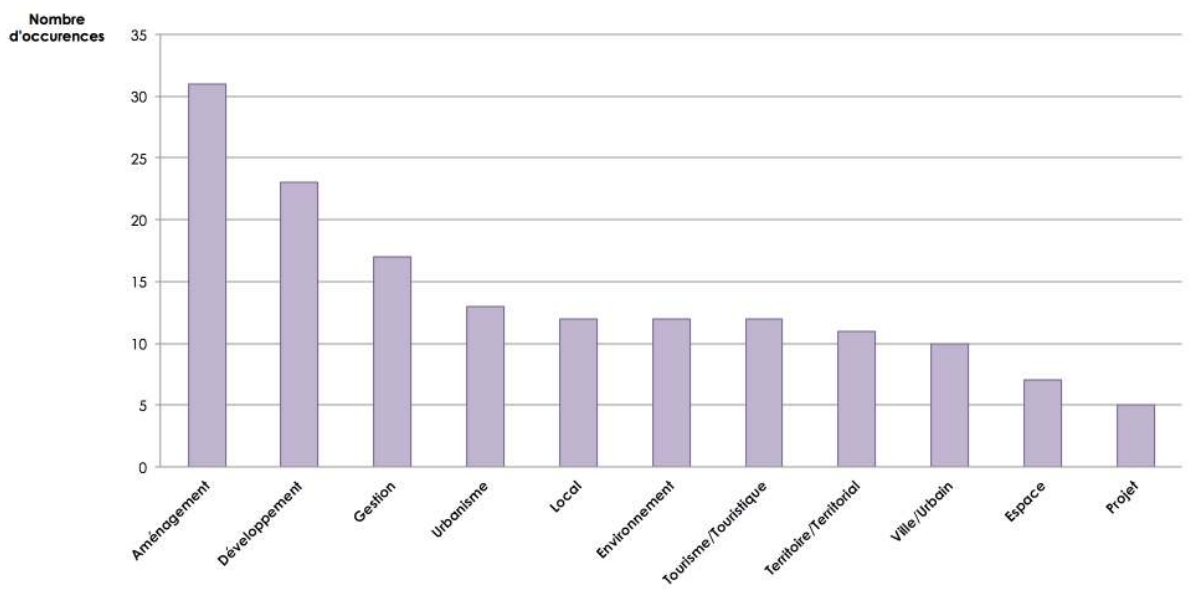

M. Pichon, 2016. Source: Bulletin Intergéo, $n^{\circ} 129$.

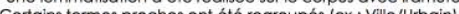

Carnets de géographes, 10 | 2017 
De façon générale, en observant seulement les DESS de " géographie et assimilés » ou de seule géographie, ces occurrences ${ }^{22}$ sont sensiblement les mêmes (voir figure 3). Pour l'essentiel, c'est donc toujours l'aménagement, le développement et la gestion qui sont les domaines les plus récurrents dans les formations délivrées au sein des DESS. On constate cependant quelques différences selon le corpus observé : le « développement » apparaît en deuxième pour l'ensemble des DESS, alors qu'il se trouve en troisième place pour les DESS de géographie et assimilés et de géographie seule. L'hypothèse que l'on peut formuler est celle d'une formation au développement - qui est souvent lié à des enjeux économiques - peut-être un peu plus prise en charge dans des départements ou UER plus éloignés de la géographie. Le tourisme et l'urbanisme, quant à eux, sont absents des termes les plus récurrents du corpus des composantes strictement géographiques (ils y apparaissent moins de 3 fois chacun) : à quelques exceptions près, leur enseignement est généralement assuré dans le cadre de départements ou instituts pluridisciplinaires ou non-géographiques.

Figure 3. Termes les plus récurrents dans les intitulés de DESS
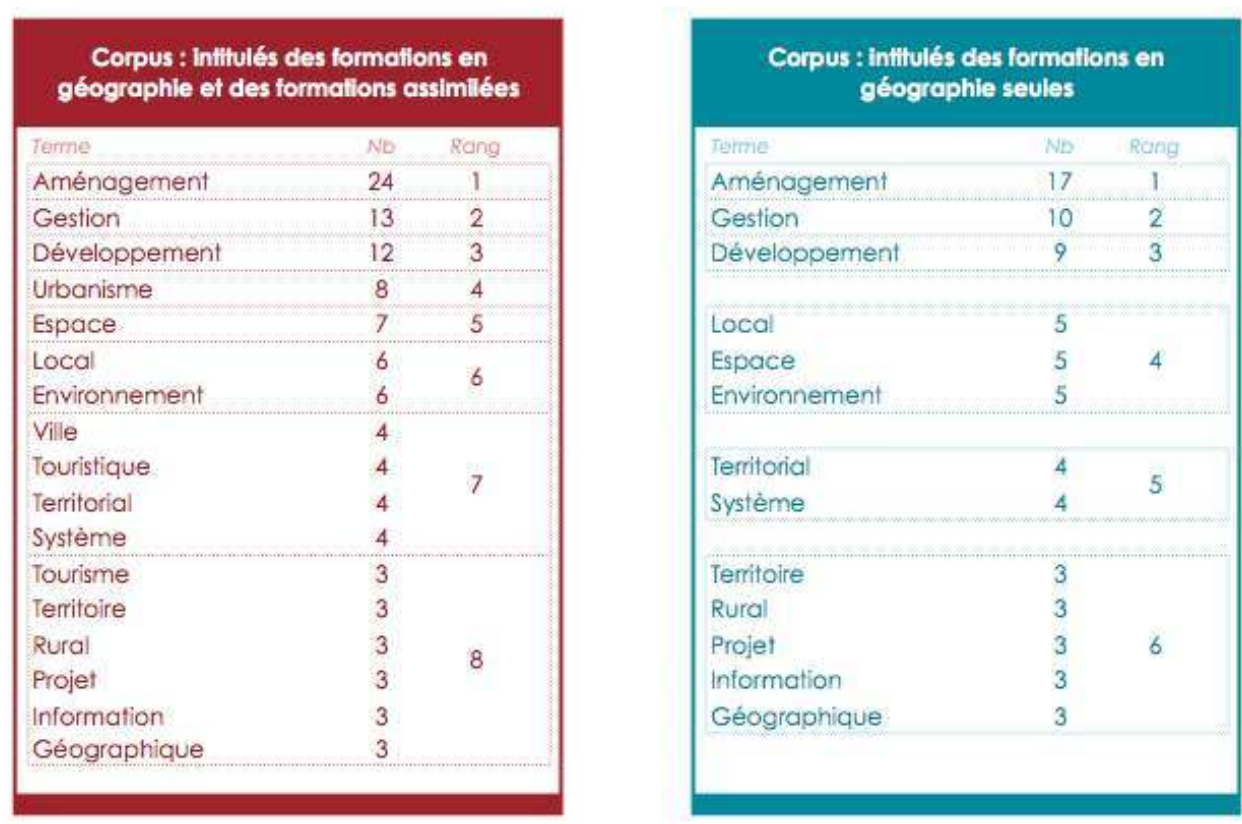

NB: dans ces tabieaux, riont été conservés que les termes qui apparaissent plus de 2 fois dans les intituiés

Enfin, en recodant le corpus pour croiser débouchés et intitulés au sein de catégories plus générales (voir annexe 2), les résultats sont là encore sensiblement les mêmes : plus de la moitié des DESS visent des débouchés dans l'aménagement, environ un tiers dans la gestion, le développement local et l'urbanisme et environ un quart dans l'environnement. 
Figure 4. Principaux domaines d'emploi visés par les DESS (géographie et assimilés) en 1998

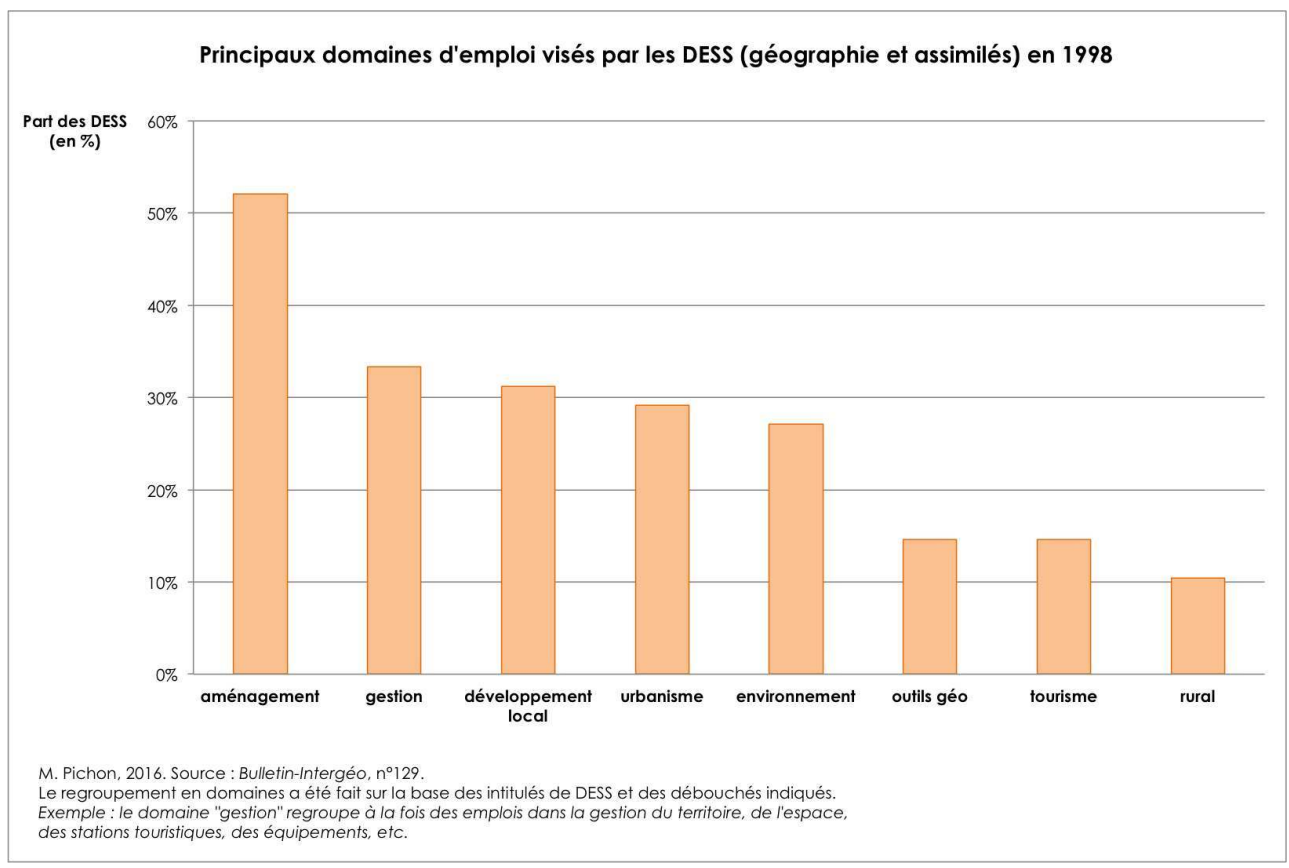

\section{Débouchés des DESS}

L'observation des débouchés détaillés tels qu'ils sont précisés dans le Bulletin-Intergéo permet d'affiner ces perspectives, en insistant non plus seulement sur les domaines visés mais aussi sur les «lieux» ou types d'emplois. Pour ce faire, nous nous sommes principalement focalisés sur les DESS en "géographie et assimilés ", afin de mettre à l'écart les formations trop éloignées de la discipline tout en maintenant une conception assez large de cette dernière. 
Figure 5. Termes les plus représentés dans les débouchés (1998)

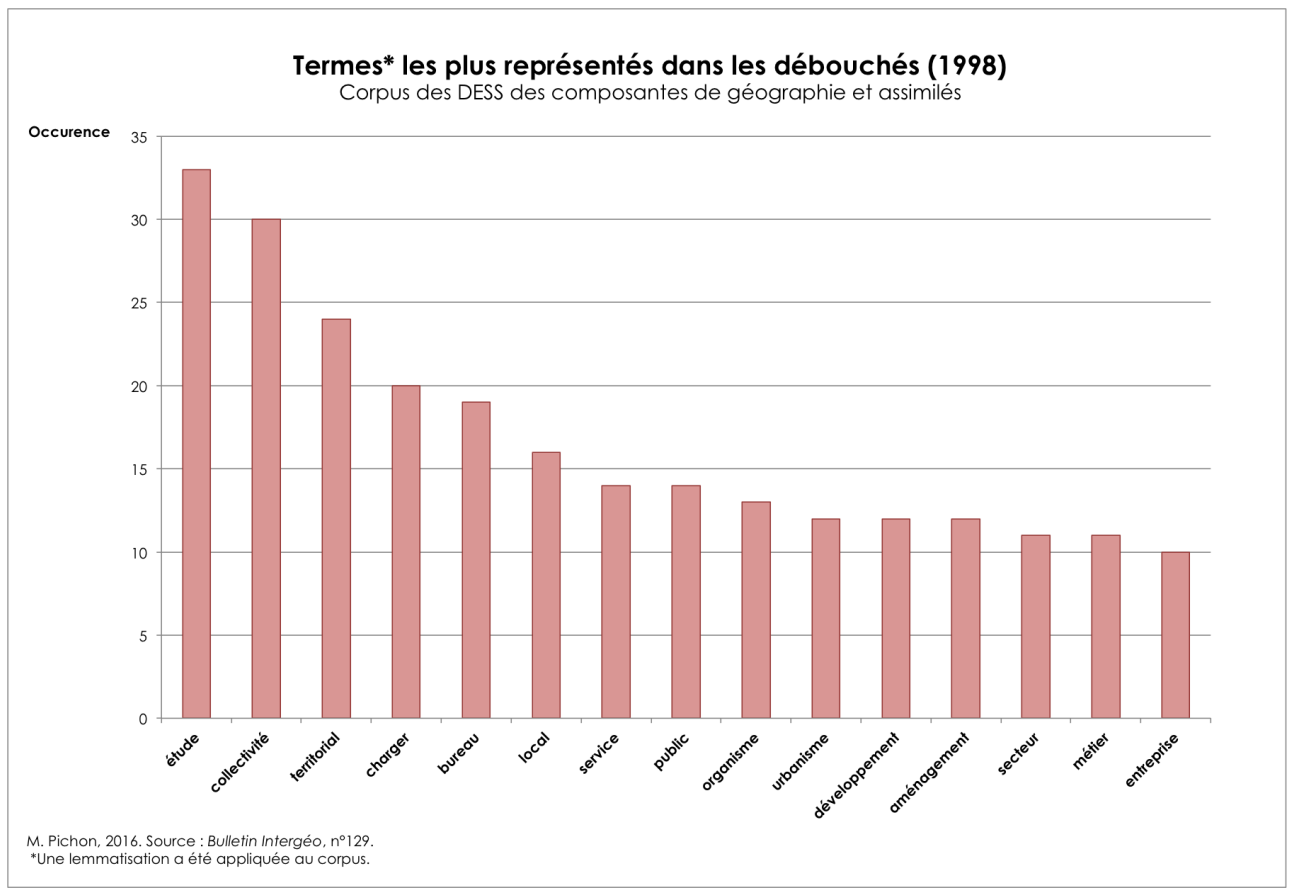

Figure 6. Analyse des co-occurrences lexicales dans les débouchés de géographie et les formations assimilées (1998)

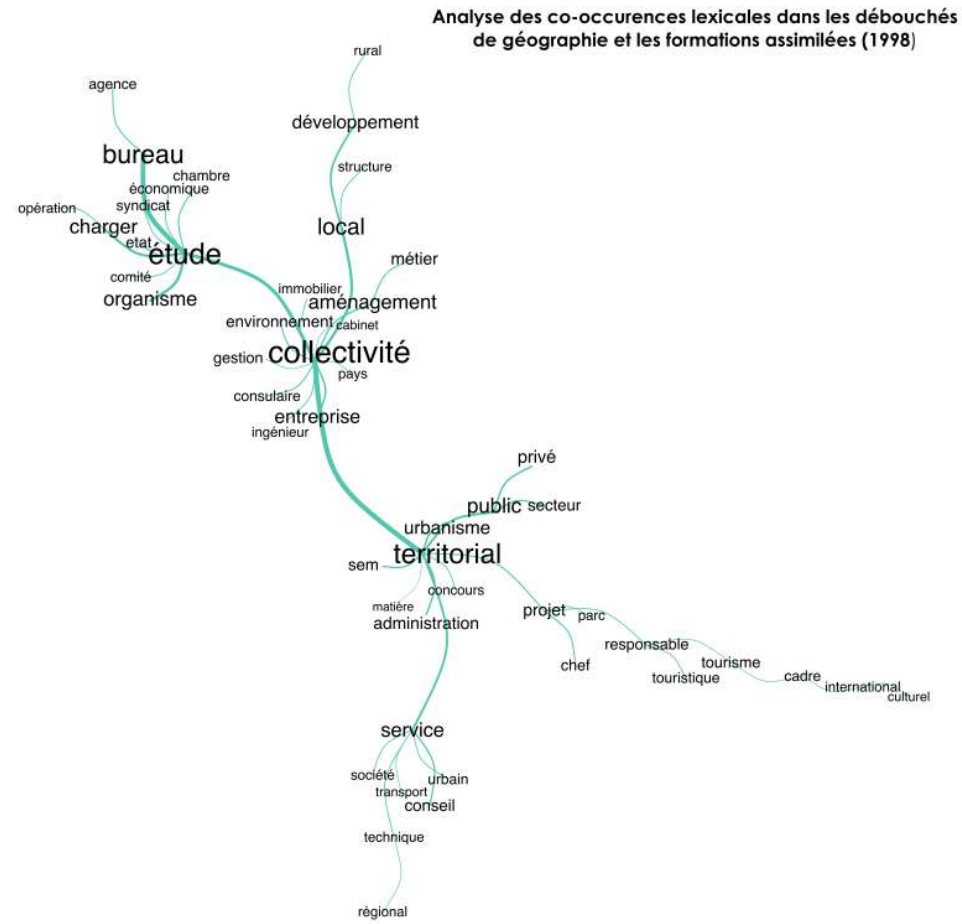

Le graphe ci-dessus présente une analyse de similitude à partir du corpus des débouchés des formations en géographie et des formations assimilées. Il est réalisé à partir d'un indice de cooccurrence : des liens épais entre deux termes signifient que ces termes sont souvent associés dans le corpus. Quant à la taille des termes, elle est proportionnelle à leur fréquence au sein de ce dernier. Là encore, le corpus a été lemmatisé. 
L'analyse lexicométrique des débouchés permet de dégager deux traits principaux: l'échelle des emplois visés par les DESS de géographie semble être celle du "territoire ", essentiellement local ou régional, et cette action sur le territoire se fait plutôt au sein des collectivités locales ou de bureaux publics ou privés. Il semble que le domaine des " études » soit dominant, avec une forte récurrence des emplois de " chargés d'études ». Il semble d'ailleurs faire le lien entre le monde des «bureaux » et celui des « collectivités ", puisqu'on le retrouve associé à ces deux secteurs d'emploi. De façon générale, la géographie "professionnelle » (en tout cas celle des DESS) semble donc s'intégrer dans le champ de l'action publique (et, dans une moindre mesure privée) territoriale et locale. Les fonctions visées semblent mobiliser les connaissances théoriques et pratiques acquises au cours du DESS : sans être toujours strictement «techniques» (cartographie, analyse de données, télédétection...), ni réellement décisionnelles, ces fonctions paraissent s'inscrire dans une sorte d'entre-deux, le champ de «l'étude ", qui peut relever du «versant cognitif » de l'action publique, c'est-à-dire l'ensemble des «éléments de connaissance, des idées » qui rentrent dans «l'élaboration des politiques publiques » (Surel, 2014).

En somme, ces quelques analyses nous permettent d'apprécier les types de débouchés visés par les DESS de géographie (et les DESS proches). Il est frappant de constater la relative stabilité de ces débouchés entre les années 1970 et les années 2000: dans l'ensemble, les domaines visés restent les mêmes, mêlant aménagement, gestion de l'espace, développement local, urbanisme ou encore environnement (ce dernier semble plus récent, cependant). Quant aux types d'emplois, il s'agit toujours principalement des organismes, administrations et bureaux en lien avec l'action publique locale ou territoriale. Les évolutions se font donc moins au sein des types et domaines visés ou occupés par les géographes "professionnels » que dans les proportions prises par cette "professionnalisation »: en effet, si ces marchés d'emplois changent peu, en revanche la place qu'ils occupent dans l'ensemble des débouchés des formations de géographie tend à s'accroître, notamment grâce au développement de formations dédiées - DESS, mais l'on pourrait aussi parler des MST, ou encore de certains DU - qui se multiplient à partir des années 1990. Ainsi, le mouvement de "professionnalisation » en géographie (entendu comme ouverture vers des emplois hors de l'enseignement) semble plutôt se faire sur un versant quantitatif (développement, multiplication, part croissante du «hors enseignement») que sur un versant qualitatif (stabilité des types d'emplois et des domaines visés ou occupés).

\section{La « professionnalisation » dans la géographie et l'aménagement aujourd'hui : un marqueur disciplinaire?}

\section{Le cas de Paris-7 (Paris-Diderot)}

Dans les années 1990, la position de la géographie au sein de l'Université Paris 7 évolue. Alors que la proximité avec les historiens était forte avec des parcours souvent bi-disciplinaires et des circulations d'étudiants entre les disciplines et entre les différents cycles universitaires, les deux départements prennent progressivement plus de distance. En effet, la préparation aux concours devient 
moins importante pour les géographes et les autres formations dites « professionnelles » se développent notamment dans le cadre d'un rapprochement avec les économistes pour développer un master commun sur « les études et le conseil » qui vient intégrer la filière en aménagement (de Félice, 2009) qui s’éloigne donc de la géographie. De plus, le rapprochement avec les économistes se poursuit actuellement avec la création d'une double licence économie-géographie (dans le cadre des financements de l'Idex Sorbonne-Paris-Cité) qui a débuté à la rentrée 2016. Par ailleurs, un projet de double licence géographie-sciences de la terre pourrait lui aussi voir le jour dans les prochaines années grâce aux mêmes financements. Cette diversification s'effectue alors que la licence majeur-mineur ou mineur-majeur entre la géographie et l'histoire décline du point de vue de son nombre d'étudiants (1 étudiants en L3 en majeur géo / mineur histoire et entre 20 et 30 en majeur histoire / mineur géo).

Aujourd'hui, au niveau du 2e cycle, le master de Géographie et sciences des territoires (GST) de Paris-Diderot comporte depuis 2014 actuellement différentes spécialités :

Carthagéo (outils et géomatique)

Espaces et milieux (commune aux mentions des masters GST et Biogéosciences)

Dynamique des milieux et des risques (DYNARISK)

Télédétection et géomatique appliquées à l'environnement (TGAE)

Dynamiques des Pays émergents et en Développement

Dynamiques spatiales en Europe et savoirs géographiques (DESG)

La filière en aménagement est distincte de celle de la géographie. Ainsi le Master Métiers des études, du conseil et de l'intervention (MECI) est commune aux économistes. Cette formation de Master 2 en Aménagement et développement local est l'héritière d'un DESS créé en 1977 qui reconnaissait ainsi l'importance des niveaux communal et intercommunal dans l'étude et la gestion des changements sociaux et territoriaux. Initialement porté par les acteurs de territoires laissés pour compte de la croissance, le développement local est devenu depuis un référentiel central pour la compréhension et une meilleure maîtrise du changement social ainsi que pour la rénovation des politiques publiques, de plus en plus décentralisées et articulées aux territoires.

En 2014, le gouvernement décide de réformer la nomenclature des diplômes de licence et de master qui comptait plusieurs milliers de mentions différentes avec l'ambition de la rendre plus simple et plus lisible pour les étudiants. Pour la licence (arrêté du 22 janvier 2014 $4^{23}$ ) la géographie est associée à l'aménagement quelle que soit l'importance de l'aménagement dans la formation, ce qui explicite et accentue l'association entre les deux et le fait que l'aménagement est de plus en plus considéré comme un débouché naturel pour les géographes. Pour les masters (arrêté du 4 février $2014^{24}$ ), la géographie et l'aménagement se retrouvent dans 7 mentions différentes : 
Géographie ;

Géographie, aménagement, environnement et développement ;

Géopolitique ;

Architecture, urbanisme, paysage ;

Géomatique ;

Gestion des territoires et développement local ;

Etudes du développement.

Par ailleurs, les formations de master ne doivent plus marquer la distinction entre masters « professionnels » ou « recherche » qui reproduisait l'ancienne division entre diplômes d'études approfondies (DEA), menant à la thèse, et diplômes d'études supérieures spécialisées (DESS), préparant au marché du travail. Les masters deviennent « indifférenciés » et préparent donc à l'une et/ou l'autre des finalités par le biais de parcours diversifiés comportant une initiation à la recherche et une part de " professionnalisation ». Enfin, les formations doivent expliciter un référentiel de compétences qui se situe à « l'interface du monde universitaire et du monde socioéconomique ». Ces documents s'adressent aux étudiants mais aussi au monde professionnel et doivent permettre une compréhension mutuelle entre ces univers : «Ils doivent ainsi permettre aux diplômés de valoriser leurs acquis, représentatifs du niveau de qualification correspondant à la licence. Référentiels de compétences et non de formation, ils n'ont pas vocation à définir des contenus d'enseignement, des volumes horaires ni des méthodes pédagogiques, qui relèvent naturellement de l'autonomie des établissements et de la liberté pédagogique de leurs enseignants. En revanche, ils définissent des objectifs de formation, exprimés en termes de compétences à acquérir tout au long de la formation. $»^{2526}$ Ces référentiels participent donc à la mise en avant de l'insertion professionnelle comme étant un des deux objectifs de la licence (avec la poursuite d'études). Par ailleurs, l'élaboration de ce document doit faire l'objet d'une large concertation incluant notamment les branches professionnelles et les partenaires sociaux.

La préprofessionnalisation des formations en géographie s'est traduite d'abord et avant tout par un fort développement de formations en aménagement et en urbanisme et s'est faite en écho à des débats épistémologiques forts. Cela s'est aussi traduit en 2009 en licence par des enseignements de « pré-professionnalisation » sur les métiers des géographes, qui sont développés lors des deux premières années de licence. En première année, en cours magistral, une série de conférences associent des enseignants et des anciens élèves qui viennent présenter leurs domaines d'activités (aménagement, enseignement, développement, cartographie et SIG, géomarketing, environnement...). En TD (UE Pré-professionalisation), les étudiants réfléchissent à leur parcours à venir en explicitant le choix des options durant les trois années de la scolarité de licence afin d'affiner un projet professionnel. Ensuite, en deuxième année, ils explicitent un projet d'insertion professionnelle plus précis et se préparent à la recherche d'un stage ou d'un emploi. Pour cela, ils s'initient à la recherche et à l'analyse d'annonces d'emploi, rédigent un CV et une lettre de motivation et s'entraînent à l'entretien d'embauche. Au-delà de ces enseignements, de nombreux enseignants se mobilisent dans une réflexion sur l'éventail des débouchés. Grâce à un partenariat avec le département de Cinéma une série de 18 
films présente des portraits d'anciens élèves, ainsi qu'une table ronde plus générale qui insiste sur les enjeux d'insertion professionnelle et de stages. ${ }^{27}$

\section{La " professionnalisation » : un enjeu des études en géographie à Paris Diderot?}

Nous avons réalisé une enquête par questionnaire en septembre 2015 lors de la première semaine de cours, auprès des étudiants inscrits en première année de licence de géographie, de majeur histoire/mineure géographie ou de MIASHS ${ }^{28}$ à l'Université ParisDiderot. Ce questionnaire visait à identifier les représentations que les étudiants ont de la géographie, ce qui a motivé leur choix d'orientation et leur vision des débouchés professionnels du cursus qu'ils ont choisi. Cinq questions leur ont été posées :

- Donnez 3 termes que vous associez à la géographie.

- Quelle définition donneriez-vous à la géographie?

- D’après vous, à quoi sert la géographie?

- Quels métiers associez-vous à la géographie?

- Pourquoi avez-vous choisi un cursus en géographie?

Le questionnaire comprenait aussi un volet permettant de cerner les caractéristiques scolaires des étudiants. 94 quatorze étudiants ont répondu à l'enquête mais 82 seulement répondent aux critères d'anonymat fixés. Les étudiants justifient d'abord leur choix d'orientation par goût ou intérêt pour la géographie ou certains de ses objets, notamment l'environnement, le développement durable ou l'aménagement. C'est un argument cité par $60 \%$ des étudiants et qui représente $49 \%$ des arguments cités ${ }^{29}$. C'est ce que montre la figure ci-dessous.

Figure 7. Justifications données par les étudiants pour le choix de leur orientation

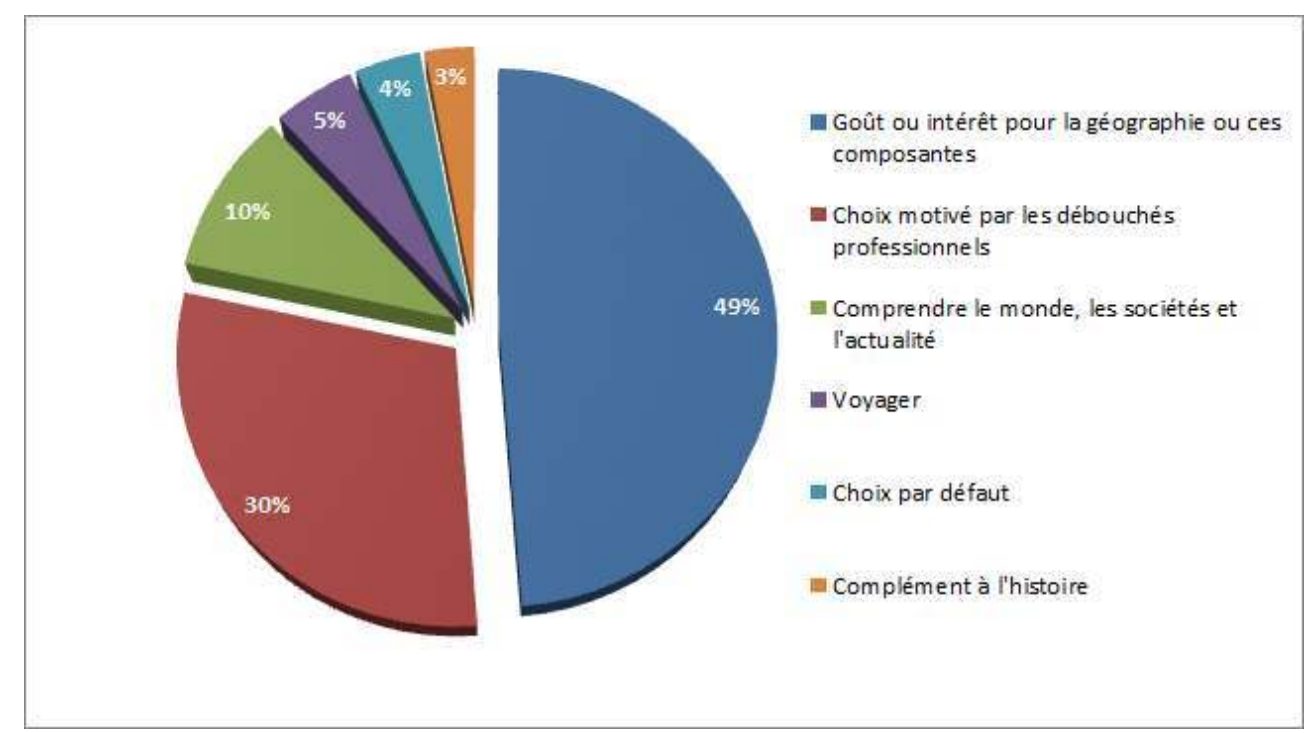

SOURCE : ENQUÊTE U. PARIS-DIDEROT,UFR GHES, 2015.

Les débouchés professionnels apparaissent seulement en seconde position, cités par $37 \%$ des étudiants, ce qui représente $30 \%$ des arguments avancés. Ces résultats diffèrent 
sensiblement de l'enquête menée par Saeed Paivandi (2011) à partir d'un corpus d'entretiens auprès de 115 étudiants, inscrits entre 2005 et 2008 dans différentes disciplines de sciences humaines et sociales et de diverses origines sociales. Le projet professionnel des étudiants y apparaît comme la logique prédominante dans leur choix d'orientation, avec $44 \%$ des étudiants «qui justifient leur choix par une attente professionnelle et l'apprentissage d'un métier. Ils visent une formation leur permettant de trouver un emploi à la fin de leurs études " (Paivandi, 2011, § 23). Inversement, seulement $20 \%$ des étudiants y mettent en avant l'intérêt ou le goût pour la discipline. Les différences entre les deux recherches peuvent s'expliquer par la méthodologie: les entretiens apportent des réponses plus riches s'il est possible d'approfondir en relançant l'interviewé si besoin. Les écarts s'expliquent probablement surtout par les différences entre les populations enquêtées: d'un côté, des étudiants de L2, L3 et master, suivant diverses discipline de SHS et inscrits dans plusieurs universités ou d'autres structures d'enseignement supérieur (écoles, IUT, etc.) réparties sur toute la région parisienne; de l'autre uniquement des étudiants de première année de géographie à Paris-Diderot. La population étudiante enquêtée à l'université Paris-Diderot vient à $87 \%$ de baccalauréat de filières générales alors que la proportion dans l'échantillon de Saeed Pavendi est de $50 \%$. Cela découle de l'origine scolaire et sociale des étudiants parisiens, généralement plus favorisés que ceux des universités périphériques. Or, le choix d'un cursus fondé sur l'intérêt ou le goût pour une discipline - ce que Saeed Paivandi appelle le "projet intellectuel» des étudiants - concerne de manière privilégiée les étudiants issus de catégories aisées. La surreprésentation d'étudiants qui choisissent la géographie à ParisDiderot parce qu'ils aiment cela s'expliquerait donc plutôt par l'origine sociale des étudiants.

En revanche, les grands débouchés professionnels sur lesquels peuvent aboutir un cursus en géographie sont relativement bien identifiés par les étudiants comme le montre la figure ci-dessous.

Figure 8. Métiers sur lesquels peuvent déboucher un cursus en géographie d'après les étudiants interrogés

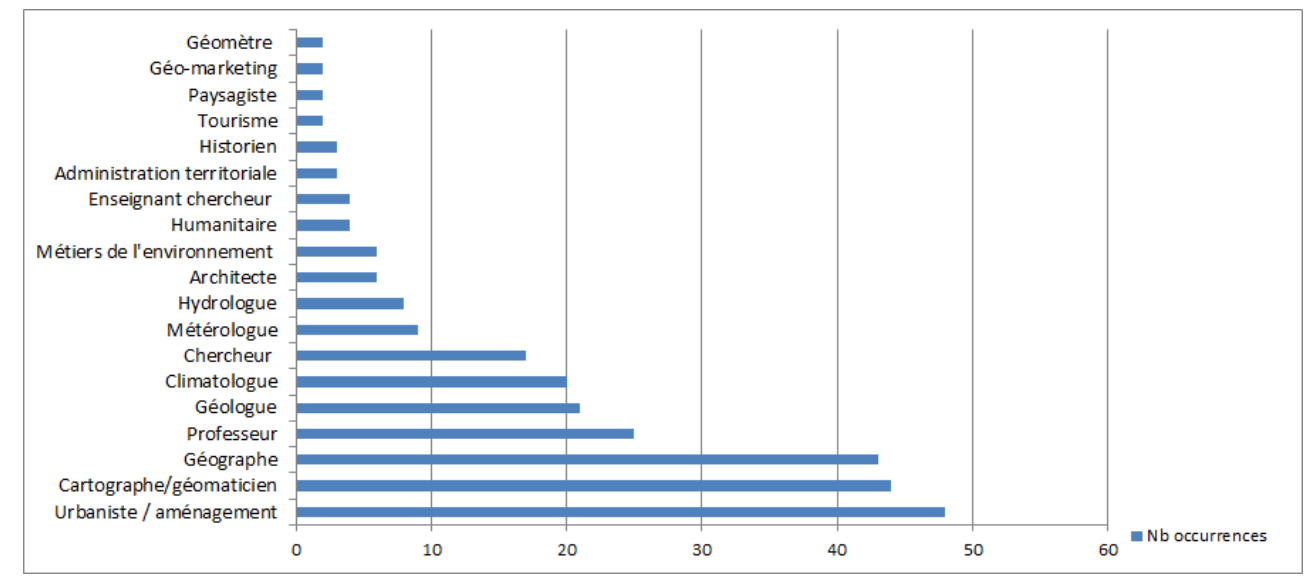

SOURCE : ENQUÊTE U. PARIS-DIDEROT, 2015.

61 Parmi les débouchés cités figurent d'abord l'urbanisme et l'aménagement ainsi que la géomatique, puis viennent, mais nettement moins cités, l'enseignement, les métiers de l'environnement et la recherche. Néanmoins, la limite de ces grands domaines professionnels n'est pas toujours claire pour les étudiants qui associent à la géographie 
les métiers de géologue, météorologue, architecte ou géomètre. Ce sont des métiers proches de la géographie mais qui nécessitent d'intégrer et de suivre d'autres cursus de l'enseignement supérieur. Enfin, « géographe » est très souvent cité comme un métier en soi sans que par ailleurs les étudiants parviennent à bien définir ce dont il s'agit. La définition demandée de la géographie oscille d'ailleurs entre un champ de connaissances et une science : « étude » (42 citations), « science » (22 citations), « matière » (3 citations), « discipline » (2 citations), « connaissance » (1 citation).

Pour conclure, les débouchés professionnels des études en géographie sont globalement connus des étudiants interrogés au début de leurs cursus mais pas de manière précise. Nous ne pouvons pas dire à l'issue de l'enquête que la géographie, pour les étudiants, se distingue des autres sciences humaines par sa forte professionnalité. En revanche, du point de vue des enseignants, la " professionnalisation » semble constituer une dimension forte au sein de la discipline et de son enseignement.

\section{Des pédagogies associées à la professionnalisation en géographie}

Dans les différentes corpus d'entretiens réalisés, les enseignants-chercheurs associent la "professionnalisation " des formations en géographie et en aménagement à trois modalités pédagogiques spécifiques : le recours à l'étude de cas, l'organisation d'ateliers et les stages ou sorties de terrain. Nous excluons ici de notre analyse les UE préprofessionnelles dont la mise en place a été traitée précédemment et qui ne sont pas propres à la discipline. Notre analyse s'appuiera ici sur nos corpus d'entretiens et sur l'analyse des brochures des formations en géographie proposées à l'Université ParisDiderot $^{30}$.

\section{L'étude de cas, copie d'une pratique professionnelle}

Le recours au cas dans les cours d'aménagement ou d'urbanisme s'inscrit dans une perspective professionnelle :

"C'est sûr qu'en aménagement c'est presque impossible de faire des cours sans utiliser des exemples ou des études de cas parce que quand même le bout du bout de l'enseignement en aménagement et urbanisme c'est de faire en sorte que les étudiants soient des professionnels avisés vis à vis des questions concrètes d'aménagement. On est quand même toujours obligé de partir d'un certain nombre de situations concrètes qui leur sont expliquées pour les mettre en situation » (entretien avec un enseignant-chercheur en aménagement).

Le cas est une mise en situation proche de celle du milieu professionnel à partir de projet d'aménagement réel. Mais l'analyse des plaquettes des formations montre que l'enseignement par cas ne se limite pas à l'aménagement. Dès 1972, l'étude de cas apparaît comme une démarche pédagogique employée en analyse spatiale, en biogéographie, en géomorphologie et en géographie régionale. Ce sont des cas «concrets» ou "d'application » qui semblent s'inscrire souvent dans une démarche déductive : c'est une application ou une illustration des apports théoriques présentés préalablement. Les entretiens que nous avons menés auprès d'enseignants-chercheurs sur leur usage des cas conduisent au même constat : 
"L'étude de cas va être, pour moi, [...] une étude approfondie autour d'un exemple, encore une fois, pour illustrer une notion, un chapitre... Pour moi, l'étude de cas arrive dans les travaux dirigés et les exemples sont donnés de façon ponctuelle et dans les $\mathrm{CM} »$ (entretien avec un enseignant-chercheur en aménagement). géographie empirique s'est construite autour d'études de cas régionaux. Il y a aussi un usage inductif des cas qui précédent alors le cours, notamment en aménagement. Finalement, si le lien entre étude de cas et "professionnalisation » est clair dans le discours des enseignants-chercheurs en aménagement, ce n'est pas le cas dans d'autres champs de la géographie qui mobilisent plus la notion d'« exemple » (Leininger, Douay et Cohen, 2016). Les études de cas peuvent être réalisées en classe à partir d'un corpus de documents. Elles peuvent aussi se construire à partir d'observations de terrain, lors de sorties, de stages collectifs ou encore d'ateliers.

\section{Les ateliers}

67 L'organisation d'ateliers est une modalité pédagogique qui semble réservée aux formations de deuxième cycle : maitrise, DESS puis master. Le terme d'atelier n'apparaît pas dans les brochures de DEUG et de licence analysées à Paris-Diderot. Les ateliers sont mentionnés dans les cours de photo-interprétation / télédétection, d'aménagement du territoire, de méthodologie ou encore dans la perspective de préparation d'un stage de terrain depuis la création de ces cours. Nous avons peu d'éléments pour préciser la mise en œuvre de l'atelier dans ces différents cours. Il est probable que le terme renvoie à des modalités pédagogiques hétéroclites. En aménagement, l'atelier vise à produire une réponse à une commande réelle ou simulée, publique ou privée, en matière d'aménagement. Les ateliers reposent sur une pédagogie de projet en petits groupes. La démarche vise à reproduire les pratiques professionnelles et leurs conditions d'exercice en collectivités territoriales, cabinets d'étude ou agences d'urbanisme qui servent de pratiques sociales de références (Martinand, 1986). Il s'agit de s'approprier et d'appliquer des savoirs et des savoir-faire mais aussi de développer une posture réflexive pour prendre de la distance par rapport aux tenants et aboutissants de la pratique professionnelle. Le lien entre atelier et "professionnalisation » est alors explicite tant dans le discours des enseignants-chercheurs qui mettent en œuvre ces ateliers que dans les brochures analysées. Par exemple, la brochure du DESS « Aménagement, animation et développement local » de Paris-Diderot stipule, en 1996-1997 :

« Le DESS propose, en un an, une formation de 3ème cycle théorique et pratique, à finalité professionnelle. L'objectif des enseignements, ateliers et stages est de :

- rendre capable d'effectuer des diagnostics,

- mettre en œuvre des stratégies,

- monter des projets,

- évaluer les programmes et les actions menées,

en insistant sur le développement des capacités d'expression, d'initiative et

d'organisation des habitants et citoyens. Au delà des compétences, cela nécessite de 
renforcer les aptitudes à l'écoute, à l'accompagnement des porteurs de projets, à la médiation et à la négociation. » raisons. Cela tient d'une part à la nature de l'aménagement qui se définit autant, si ce n'est plus, comme une pratique que comme une discipline professionnelle. D'autre part, cela tient aussi aux exigences du réseau des écoles d'urbanisme francophone de l'APERAU qui labellise les formations après un audit. En effet, l'acte fondateur de l'association renvoie à l'adoption d'une "charte enrichie tout au long des 30 dernières années par des discussions avec des partenaires publics (ministères en charge de l'urbanisme ou de l'enseignement supérieur) et les organisations de la profession et le débat entre institutions universitaires de différents pays » (site web de l'APERAU) ${ }^{31}$. Le contenu de la charte fait donc référence à «la construction d'une culture commune » en aménagement et à " une modalité de travail collectif du type de l'atelier professionnel, de préférence sur commande ». Plus concrètement, la réponse à des ateliers correspond aussi à un principe de réalité financière dans un contexte de restriction des ressources budgétaires. En effet lorsque la commande donne lieu à une rémunération cela peut constituer une ressource importante pour l'organisation d'un voyage d'études, l'achat de matériel ou la rémunération d'intervenants extérieurs. Dès lors, au-delà de l'intérêt pédagogique, il peut parfois se développer une dépendance à ces commanditaires et une nécessité de répondre le plus efficacement possible pour fidéliser le commanditaire et son financement, ce qui n'est pas sans poser de questions sur la nature critique de la réponse à cette commande.

\section{Le terrain}

Troisièmement, l'enseignement en dehors du campus, "sur le terrain", semble aussi constituer une caractéristique forte de l'enseignement de la géographie et de l'aménagement et correspond notamment à un objectif plus général de "professionnalisation ${ }^{32}$. Dans ce sens le terrain pourrait être une des spécificités de la géographie :

« Une difficulté est qu'ils ne sont pas là par choix, il faut leur montrer que l'objectif de l'enseignement supérieur est différent, que c'est un objectif professionnalisant; c'est souvent par le biais du contact avec le terrain qu'ils perçoivent la réalité de ce que peut être le métier de géographe, et que finalement on arrive à faire éclore des vocations » (entretien avec un enseignant-chercheur de Paris Diderot, 2013).

Le terrain est le lieu d'une pratique professionnelle. C'est aussi un lieu et un temps de rencontre avec des professionnels, urbanistes, chargés de mission au sein de parcs naturels, etc. Le terrain peut prendre plus ou moins d'importance dans la temporalité des pratiques pédagogiques. Pour le cas de l'Université Paris-Diderot, la référence au «terrain » est récurrente et apparaît dès les premières plaquettes de présentation du cursus en géographie en 1971. Le terrain participe à cette idée d'une pratique de la géographie à Paris-Diderot qui serait plus critique et plus ouverte sur la diversité des espaces et des sociétés ${ }^{33}$. C'est un des éléments constitutifs de l'identité revendiquée du département ou en tout cas représenté comme tel par les collègues de cette université. Ce fort attachement au terrain est prégnant dans le discours des enseignants-chercheurs du département de géographie mais aussi dans celui des étudiants. L'UNEF a par exemple fait parvenir une motion au Conseil des études et de la vie universitaire du 15 juin 1989 pour défendre l'idée selon laquelle les stages de terrain en géographie «sont reconnus comme 
une spécificité de l'enseignement de la géographie à Paris 7 (rapport national d'évaluation). Ces stages permettent aux étudiants d'appliquer, face aux problèmes concrets, les connaissances théoriques acquises en salle. Ces stages permettent l'analyse des liens existants entre milieu naturel et activités humaines et sont l'occasion de fructueux échanges multidisciplinaires, une des vocations de notre université. Ces stages sont appréciés des étudiants et de leurs employeurs qui voient là un atout supplémentaire à leur formation.» Le lien entre le terrain et la "professionnalisation » est explicite. D'ailleurs, à partir de 2005, les plaquettes de présentation de la licence de géographie contiennent un paragraphe introductif qui mentionne, entre autres, le terrain comme levier de "professionnalisation ", au côté des outils techniques :

«A travers les thématiques abordées [...], les méthodes de travail employées (travaux de terrain par petits groupes et études de cas permettant la confrontation aux réalités physiques et sociales, travaux de laboratoire...) et l'utilisation des outils modernes d'investigation [...], les enseignements de la mention "Géographie" de l'Université de Paris-Diderot / Paris 7 cherchent à concilier l'acquisition indispensable d'un socle de connaissances fondamentales et le développement de compétences techniques. Ce volet appliqué de l'enseignement, qui donne un caractère professionnalisant indéniable aux études de géographie, est également une réponse à la demande sociale »

71 Du point de vue des pratiques pédagogiques, le terrain peut prendre plusieurs formes. En effet, la sortie peut être ponctuelle par exemple dans le cadre d'une séance de TD. Cela peut donner lieu à l'organisation d'un "stage de terrain » qui s'apparente à un voyage d'étude qui permet la mise en application des enseignements sur le terrain. Le stage va souvent donner lieu à une préparation pendant tout un semestre puis un déplacement d'une ou deux semaines permet de mettre en application les enseignements par l'observation, la collecte et l'analyse d'informations. "Une sortie sur le terrain permet de mettre en pratique les acquis de GO 230 (Types d'espace) et de les relier à cet enseignement ", mentionne Laurent Faret (professeur en géographie) dans la plaquette 2002-2003 de la licence de Géographie de Paris-Diderot. Le stage de terrain est souvent seulement mentionné dans la description des cours faite dans les plaquettes des formations. Le lien avec la « professionnalisation » n'est pas explicite à cet endroit. Il y a pourtant une forte relation entre la montée en puissance de l'affichage de la «professionnalisation » de la licence et la mention du terrain comme démarche pédagogique dans les enseignements, comme le montre le graphique ci-dessous. 
Figure 9. Nombre de références au terrain et au monde professionnel dans les fascicules des formations de géographie analysés de l'Université Paris-Diderot (1972-2012)

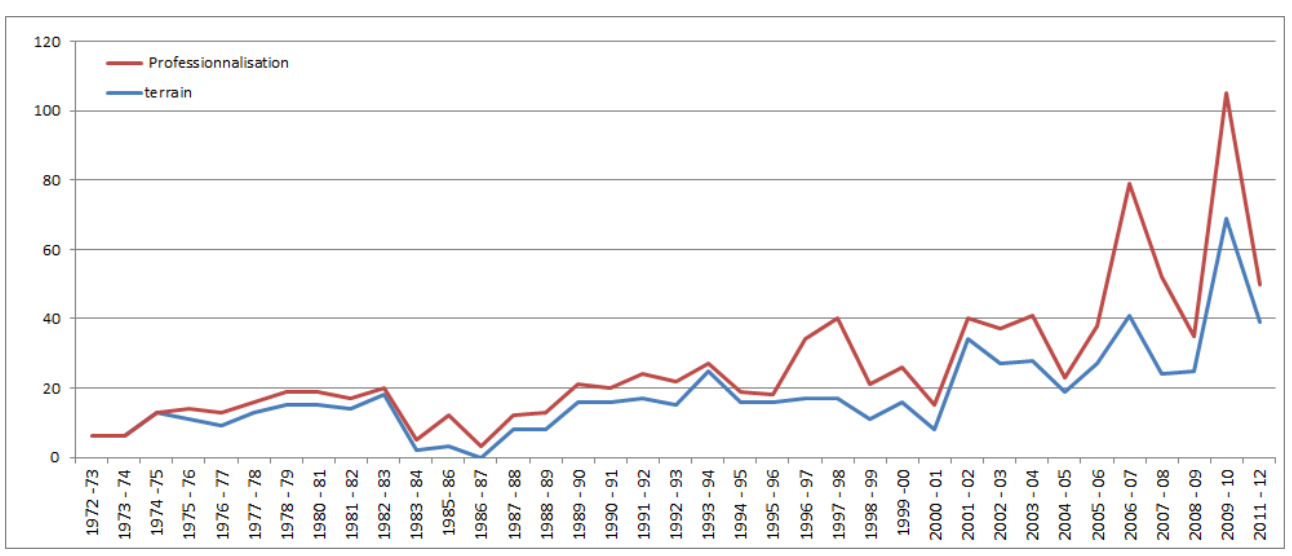

SOURCE : ARCHIVES U. PARIS-DIDEROT

Les références à la « professionnalisation » ou au monde professionnel ont augmenté de manière continue depuis l'ouverture de l'Université Paris-Diderot avec une accélération du nombre de mention à partir du milieu des années 2000. Le nombre de mentions du «terrain" dans les brochures évolue de manière identique. Cette co-évolution (qui semble indiquer une corrélation) va dans le sens du lien établi entre le terrain et la «professionnalisation » dans les entretiens menés auprès d'enseignants-chercheurs de géographie.

\section{Conclusion}

L'enjeu de la "professionnalisation » apparaît finalement comme étant au cœur des transformations pédagogiques de la géographie universitaire depuis les années 1970. L'hypothèse selon laquelle cette mutation correspondrait à un double mouvement ascendant et descendant nous semble à nouveau pertinente.

La logique descendante renvoie au champ institutionnel et politique qui, par une série d'injonctions, élabore progressivement une série de normes qui doivent s'appliquer à l'ensemble du champ universitaire : de ce point de vue, la «professionnalisation » n'est pas spécifique à la discipline. Ces normes sont plus ou moins acceptées et intériorisées par les enseignants-chercheurs en géographie alors que la discipline a connu une crise sociale et épistémologique marquante qui interroge notamment l'utilité et l'identité de la discipline. En effet, alors que l'enseignement était le débouché naturel des étudiants de géographie, les perspectives d'insertion professionnelle se sont raréfiées, conduisant alors à la redéfinition de ces mêmes perspectives. De ce fait, ces injonctions générales à la "professionnalisation » vont rencontrer un contexte disciplinaire plus endogène, ce qui contribue à questionner la fonction sociale et économique de la géographie et à redéfinir, au moins pour partie, son identité. Il s'agit alors de réaffirmer une nouvelle utilité en reformulant la demande sociale à la base de la géographie. Concrètement cette mutation se traduit par des reconfigurations progressives mais néanmoins substantielles des formations qui viennent transformer les contours de la discipline. Son objet s'est de fait élargi vers les questions d'environnement, d'aménagement et de développement. Parallèlement, de nouveaux champs académiques se sont développés (comme 
l'aménagement et l'urbanisme), issus ou non de la géographie, et qui peuvent se placer en concurrence avec elle, notamment car ils partagent des débouchés similaires.

«Professionnaliser l'université, ce n'est donc pas seulement la rendre professionnelle. Ce n'est pas seulement l'adapter au réel des professions. C'est lui faire servir des enjeux politiques, idéologiques ou économiques qui ne relèvent pas d'elle » affirme Christophe Granger (Granger, 2015) : alors que l'injonction à la "professionnalisation » et son intériorisation sont plus fortes que jamais, qu'une grande partie des cursus de géographie se sont professionnalisés, et que ce mouvement a commencé dès les années 1970, préoccupant fortement enseignants et étudiants, à quoi doit-on s'attendre pour l'avenir proche de la discipline? Quelle doit - ou devrait - être sa fonction sociale ? Quel degré d'autonomie devrait-elle conserver ou conquérir? Finalement, les débats qui animaient les «Etats généraux de la géographie » de 1968 gardent toute leur pertinence, et revêtent même une importance accrue : "quelles personnes désire-t-on former?». Voulons-nous d'une géographie qui «souscri[t] à la demande », ou bien d'une géographie capable de déterminer elle-même les finalités du savoir qu'elle dispense?

\section{BIBLIOGRAPHIE}

AFDG. (1987). Les débouchés professionnels des étudiants en géographie. Intergéo-Bulletin, (87), 73-101.

AFDG, INTERGEO, Plet, F., \& Briend, A.-M. (1988). Diplômes et formations universitaires en géographie, aménagement, urbanisme, tourisme, 1988. Intergéo-Bulletin, (90), 1-126.

Agulhon, C., \& Convert, B. (2011). Introduction : La professionnalisation des cursus, axe majeur des réformes européennes ? Cahiers de la recherche sur l'éducation et les savoirs, (Hors-série n 3), 519.

Bourdon E., Chevalier D., Cock L.D., Delacroix C., Garcia P., Leduc J., 2015, « Le CAPES d'histoire et de géographie ", Vingtième Siècle. Revue d'histoire, 126, p. 163-176.

Delacroix C., 2015, « Un tournant pédagogique dans la formation des enseignants », Annales. Histoire, Sciences Sociales, 70e année, 1, p. 191-203.

Orain O., 2006, «La géographie comme science. Quand "faire école" cède le pas au pluralisme ", dans Couvrir le monde : un grand XXe siècle de géographie française, Paris, Association pour la diffusion de la pensée française, p. 90-122.

Charlier, J.-É., \& Croché, S. (2010). L'inéluctable ajustement des universités africaines au processus de Bologne. Revue française de pédagogie. Recherches en éducation, (172), 77-84. http:// doi.org/10.4000/rfp.2276CNFG. (1972). Journées géographiques de Rennes. 10-13 mars 1972. Intergéo, Bulletin de Liaison Des Instituts et Centres de Recherches de Géographie, (27), 169-254.

Chervel A., 1993, Histoire de l'agrégation. Contribution à l'histoire de la culture scolaire, Paris, Kimé, 289 p.

CNFG. (1983). L'enseignement de la géographie dans les universités françaises, 1981-1982. Intergéo, Bulletin de Liaison Des Instituts et Centres de Recherches de Géographie, (70), 85-109. 
Collectif. (1968). États généraux de la géographie. Tours, 8-10 juillet 1968. Procès-verbaux, rapports, motions. Intergéo, Bulletin de Liaison Des Instituts et Centres de Recherches de Géographie, (12), 177-217.

Collectif. (1986). Géopratiques. Les étudiants se prennent en charge. Lettre d'Intergéo, (8).

Merlin, P. (1991). Géographie et aménagement. Travaux de l'Institut de Géographie de Reims, (79-80), 21-28.

Delacroix, C. (2015). Un tournant pédagogique dans la formation des enseignants. Annales. Histoire, Sciences Sociales, 70e année(1), 191-203.

De Félice, J., (2009), « Une histoire à ma façon : l'aménagement du territoire dans l'enseignement de la géographie », in L'Information Géographique , nº 2, vol. 73, p. 29-46.

Gayraud, L., Simon-Zarca, G., \& Soldano, C. (2011). Université : les défis de la professionnalisation - (Notes emploi formation $n^{\circ}$ 46.) (p. 36). Marseille : CEREQ. Retrieved from http:// www.decitre.fr/livres/universite-les-defis-de-la-professionnalisation-9782110981516.html

Giret, J.-F., \& Moullet, S. (2008). Une analyse de la professionnalisation des formations de l'enseignement supérieur (Net.doc No. 35) (p. 25). Marseilles : CEREQ. Retrieved from http:// www.cereq.fr/cereq/netdoc35.pdf

Granger C., 2015, La destruction de l'université française, Paris, La Fabrique, 176 p.

Leininger, C., Douay, N. \& Cohen, M. (2016), « L'étude de cas face à l'exemple : pratiques et enjeux dans l'enseignement de la géographie et de l'aménagement à l'université », Recherche en Education, $\mathrm{n}^{\circ} 27$, pp. 52-66

Martinand, J.-L. (1986). Connaître et transformer la matière. Berne : Peter Lang.

Orain O. (2006). « La géographie comme science. Quand "faire école" cède le pas au pluralisme », dans Couvrir le monde : un grand XXe siècle de géographie française, Paris, Association pour la diffusion de la pensée française, p. 90-122.

Orain, O. (2009). De plain-pied dans le monde : écriture et réalisme dans la géographie française au XXe siècle. l'Harmattan, Paris.

Orain, O. (2015). Mai 68 et ses suites en géographie française. Revue D'histoire Des Sciences Humaines , (26), 209-242.

Paivandi, S. (2011). «La professionnalisation de l'Université française : la perspective étudiante », Cahiers de la recherche sur l'éducation et les savoirs, hors-série nº 3, 167-186.

Phlipponneau, M. (1972). Une enquête sur les applications de la géographie et les débouchés pour les étudiants. In La pensée géographique contemporaine : mélanges offerts à André Meynier (pp. 87-97). Saint-Brieuc : Presses universitaires de Bretagne.

PRODIG, Briend, A.-M., \& Vélard, B. (1998). Guide des formations universitaires en géographie, aménagement, environnement, urbanisme, tourisme. Intergéo-Bulletin, (129), 1-252.

Rhein, C. (1982). La géographie, discipline scolaire et/ou science sociale ? Revue Française de Sociologie, vol. XXIII(2), 223-251.http://doi.org/10.2307/3320771

Robic, M.-C. (2006). Une discipline se construit. Enjeux, acteurs, positions. In Couvrir le monde : un grand XXe siècle de géographie française (pp. 15-52). Paris : Association pour la diffusion de la pensée française.

Stavrou, S. (2011). La « professionnalisation » comme catégorie de réforme à l'université en France. Cahiers de la recherche sur l'éducation et les savoirs, (Hors-série n 3), 93-109. 
Surel, Y. (2014). Approches cognitives. In L. Boussaguet \& P. Muller, Dictionnaire des politiques publiques (pp. 90-98). Paris : Presses de Sciences Po.

Vincens, J., \& Chirache, S. (1992). Rapport de la commission « professionnalisation des enseignements supérieurs ». Paris : Haut Comité Éducation- Économie (HCEE).

Wittorski, R. (2008). La " professionnalisation ». Savoirs, (17), 9-36.

\section{ANNEXES}

Annexe 1 Caractéristiques personnelles des enseignants-chercheurs interviewés

\begin{tabular}{|c|c|c|c|c|c|c|}
\hline Entretien & Sexe & Statut & Spécialité & $\begin{array}{l}\text { Expérience à } \\
\text { l'université }\end{array}$ & \begin{tabular}{|l} 
Expérience \\
dans le \\
secondaire
\end{tabular} & $\begin{array}{l}\text { Impliqué dans la } \\
\text { formation } \\
\text { professionnelle }\end{array}$ \\
\hline E1 & $\mathrm{F}$ & MCF & Aménagement & 7 ans & Oui & Oui \\
\hline E2 & M & MCF & Aménagement & 15 ans & Non & Oui \\
\hline E3 & M & MCF & Aménagement & 5 ans & Non & Oui \\
\hline E4 & M & MCF & $\begin{array}{l}\text { Géographie physique } \\
\text { ou } \\
\text { environnementale }\end{array}$ & 22 ans & Oui & Non dit \\
\hline E5 & $M$ & MCF & Géographie physique & 2 ans & Non & Non \\
\hline E6 & $\mathrm{F}$ & MCF & Géographie physique & 4 ans & Non & Non \\
\hline E7 & $\mathrm{F}$ & MCF & Géographie humaine & 17 ans & Non & Oui \\
\hline E8 & $\mathrm{F}$ & MCF & Géographie humaine & 20 ans & Non & Oui \\
\hline E9 & M & $P$ & $\begin{array}{l}\text { Géographie physique } \\
\text { ou } \\
\text { environnementale }\end{array}$ & 20 ans & Oui & Non dit \\
\hline E10 & M & MCF & $\begin{array}{l}\text { Géographie physique } \\
\text { ou } \\
\text { environnementale }\end{array}$ & 4 ans & Non & Non \\
\hline G1 & M & MCF & Géographie humaine & 5 ans & Non & Oui \\
\hline G2 & $\mathrm{F}$ & MCF & Géographie humaine & 10 ans & Oui & Oui \\
\hline G3 & M & MCF & Géographie humaine & 20 ans & Oui & Oui \\
\hline G4 & $\mathrm{F}$ & MCF & $\begin{array}{l}\text { Géographie physique } \\
\text { ou } \\
\text { environnementale }\end{array}$ & 7 ans & Non & Oui \\
\hline
\end{tabular}




\begin{tabular}{|l|l|l|l|l|l|l|}
\hline G5 & M & P & $\begin{array}{l}\text { Géographie physique } \\
\text { ou } \\
\text { environnementale }\end{array}$ & 25 ans & Oui & Non \\
\hline G6 & M & P & Géographie humaine & 10 ans & Non & Non \\
\hline G7 & F & MCF & $\begin{array}{l}\text { Géographie physique } \\
\text { ou } \\
\text { environnementale }\end{array}$ & 4 ans & Non & Oui \\
\hline
\end{tabular}

$\mathrm{F}=$ féminin $; \mathrm{M}=$ masculin $; \mathrm{MCF}=$ maître de conférences $; \mathrm{P}=$ professeur

Annexe 2 : regroupement et recodage des intitulés et des débouchés en grands domaines

Image 20005A1E00007A390000444211E4AF6C.wmfUne même formation peut se retrouver dans plusieurs catégories (par exemple, s'il s'agit d'aménagement touristique, on retrouvera cette formation dans la catégorie « aménagement » et la catégorie «tourisme »).

\section{NOTES}

1. Nous n'insisterons donc pas réellement sur la professionnalisation des formations à l'enseignement secondaire ou la recherche, mais plutôt sur la professionnalisation horsenseignement.

2. Voir l'entretien d'Antoine Culioli paru en mai 1990 dans le numéro spécial « Paris 7 a 20 ans » du journal de l'université 7 à dire (pages 9-11). Texte disponible en ligne : http://www.univ-parisdiderot.fr/sc/site.php?bc=archivesP7\&np=Culioli.

3. Voir le séminaire annuel de recherche du département de géographie de l'Université ParisDiderot, "Les géographies critiques à l'Université Paris-Diderot", 3 juillet 2013, organisé par N. Douay, E. Grésillon et A. Spire.

4. Le travail aux archives nationales et le dépouillement des Bulletins Intergéo s'inscrivent dans le cadre de la recherche doctorale de Matthieu Pichon. Le dépouillement des archives de ParisDiderot s'inscrit dans le cadre d'une recherche sur «La géographie et l'histoire du lycée à l'université » financé par l'Idex Sorbonne-Paris-Cité.

5. Cette enquête s'inscrit dans le cadre du projet de recherche précédemment cité.

6. Création des IUT : Institut Universitaire de Technologie en 1966.

7. Maîtrise de méthodes informatiques appliquées à la gestion (MIAGE) en 1970 ; Maîtrise de sciences de gestion (MSG) et Maîtrise des sciences et techniques (MST) en 1973 ; Diplôme d'étude supérieure spécialisée (DESS) en 1974 ; création des écoles ingénieurs dans la première moitié des années 1970.

8. Mise en place des Magistères et des Diplômes d'études universitaires de sciences et de techniques (DEUST) en 1985 et la création de diplômes spécifiques pour les Instituts Universitaires Professionnalisés (IUP) et les IUT.

9. Loi n²007-1199 du 10 août 2007

10. A l'exception d'une motion votée au CNU (23ème section) pour soutenir la bivalence au CAPES, votée lors de la session du 4 au 6 février 2014. Motion disponible sur: http:// sgencfdtamu.blogspot.fr/2014/02/cnu-23-eme-section.html. Motion réitérée par la CNU 23 en septembre 2016 
11. Notre étude historique des formations à l'échelle nationale renvoie ainsi plutôt à la professionnalisation entendue comme obtention d'un diplôme spécialisé pouvant être mobilisé dans certains marchés de l'emploi (autres que l'enseignement). Le cas de Paris-7 fait intervenir une plus grande polysémie, en mettant notamment l'accent sur le mode de formation (réalisation de stages, travail sur des cas concrets, acquisition de savoirs opérationnels...).

12. Les origines de cette crise épistémologique et sociale sont à trouver dans la «somme impressionnante de tensions, qui engagent tout à la fois le statut symbolique de la discipline, la cohésion sociale interne, les marchés auxquels ses membres ont accès et l'horizon cognitif qu'elle s'imagine » (Orain, 2009). Elle se nourrit notamment de la « dévaluation statutaire ou le sousemploi des "géographes professionnels » [expression régulièrement utilisée pour désigner les diplômés de géographie qui ne sont ni enseignants, ni universitaires], sans parler des difficultés d'accession à l'expertise des jeunes générations universitaires » (ibid.)

13. Il semble que la décennie 1970 soit, par exemple du point de vue de l'agrégation, caractérisée par « des à-coups importants dus à l'évolution des politiques scolaires : 400 postes de moins entre 1974 et 1975, effondrement du nombre de reçus entre 1977 et 1979 (de 1600 à 931), remontées tout aussi spectaculaire ensuite (Chervel, 1993).

14. Les «Journées géographiques » sont des réunions annuelles organisées par le Comité national français de géographie (CNFG), l'une des instances délibératives de la discipline. Notons que ses décisions ne font pas nécessairement autorité : c'est notamment contre le CNFG que sera créée en 1982 l'Association française pour le développement de la géographie (AFDG), autre instance de la discipline.

15. CNFG. (1975). Rapports des groupes de réflexion sur la géographie. Nice : 1-2 mars 1975. Intergéo, Bulletin de liaison des Instituts et Centres de recherches de géographie, (38), 113-164.

16. Organisées par l'association Géopratiques, qui regroupe de « jeunes géographes en formation, à la recherche d'un emploi, ou engagés dans la vie professionnelle ».

17. Elles s'expriment par exemple très régulièrement dans les commissions « Aménagement du territoire » et « Géographie appliquée » des « Journées géographiques » annuelles du CNFG.

18. Elle porte sur les inscrits en maîtrise de géographie, d'aménagement et en MST d'aménagement en 1981-1982, et donc sur le parcours universitaire et professionnel depuis ce moment.

19. Pour rappel, le laboratoire INTERGEO (devenu PRODIG) produit un certain nombre de publications, à destination de la communauté scientifique des géographes, qui visent à donner et valoriser des informations diverses (sur les recherches, enseignements, publications, thèses en cours).

20. Nous les désignerons sous la catégorie " géographie et assimilés ». Ces délimitations ne sont pas toujours aisées. Nous avons notamment inclus les instituts d'aménagement et d'urbanisme (y compris l'IATEUR de Reims, pourtant rattaché à l'UFR de droit mais lancé notamment par Roger Brunet), et les formations qui, même si elles ne relevaient pas de la géographie stricto sensu, mentionnaient dans leur intitulé la notion d' " espace " (c'est le cas notamment pour une formation en environnement et une en tourisme, qui par ailleurs ont des géographes dans leur équipe pédagogique voire dans leur direction).

21. Il s'est agi principalement de croiser intitulés de diplômes et débouchés annoncés afin de déterminer, pour chaque DESS observé, la pluralité des champs vers lesquels il prétend diriger les étudiants. Voir annexe 2.

22. Le corpus analysé a été lemmatisé : on réduit le corpus en ramenant les mots d'une même famille au lemme qui leur est commun. Par exemple, si le corpus contient les mots «territoriale", «territoriales", «territoriaux », la lemmatisation va regrouper tous ces mots sous le lemme «territorial». On peut ainsi relever plus précisément les occurrences d'un mot en faisant abstraction des diverses formes qu'il prend dans le corpus (genre, nombre, etc.).

23. https://www.legifrance.gouv.fr/eli/arrete/2014/1/22/ESRS1331573A/jo/texte 
cidTexte=JORFTEXT000028583174\&categorieLien $=$ id

25. htt p://www.enseignementsup-recherche.gouv.fr/cid61532/les-referentiels-de-competencesen-licence.html

http://cache.media.enseignementsup-recherche.gouv.fr/file/Plan_licence/61/4/ referentiel_227614.pdf,

pages 26 à 28

27. youtube.com/user/GeoParisDiderot

28. Mathématiques appliqués aux sciences humaines et sociales option géographie.

29. Les étudiants peuvent citer plusieurs arguments, les réponses étant ouvertes. Nous indiquerons donc la proportion d'étudiants ayant cité un élément et le poids de cet élément dans l'ensemble des réponses évoquées.

30. Le comptage a été opéré à partir des plaquettes de formation en géographie qui ont été numérisées par le service d'archives de l'Université Paris-Diderot : Cursus de géographie (1972 à 1982) ; Deug de géographie (1985 à 2005); Licence de géographie (1982 à 2012); Maîtrise de géographie (1987 à 2005); Master 1 GST (2006 à 2010); DESS AADL 1996 à 1998); DESS Géophysique de surface et subsurface (1993 à 1994); DESS Migrations, échanges et développement méditerranéen (1997 à 1998); Master 2 environnement, milieux techniques, société (2006 à 2007).

31. http://www.aperau.org/mission_et_objectifs_aperau/charte_aperau.html

32. Avec le "terrain », l'enseignement contemporain de la géographie réactive sans doute - sous une forme néanmoins différente, plus « professionnalisée » - ce que l'on appelait aussi autrefois «l'excursion », qui fut longtemps un "exercice-type » et un « rituel proprement initiatique et socialement intégrateur, pour les générations [d'étudiants en géographie] formées entre 1905 (date de la première excursion interuniversitaire) et la fin des années 1960 » (Orain, 2006). Cette pratique pédagogique doit ainsi être recontextualisée dans l'histoire longue de la discipline.

33. Voir le séminaire annuel de recherche du département de géographie de l'Université ParisDiderot, "Les géographies critiques à l'Université Paris-Diderot", 3 juillet 2013, organisé par N. Douay, E. Grésillon et A. Spire.

\section{RÉSUMÉS}

La géographie a connu de profondes mutations depuis les années 1970, parmi lesquelles figure la "professionnalisation» des enseignements. Celle-ci touche des domaines variés : aménagement, urbanisme, mais également environnement ou cartographie. Plus largement, ces mutations se manifestent par un double mouvement ascendant et descendant: descendant par effet de contexte et adaptation aux différentes injonctions institutionnelles; ascendant par l'existence, au sein de la discipline, de questionnements sur son utilité sociale et de débats épistémologiques alors que les débouchés dans l'enseignement secondaire se tarissent. Plus concrètement, cela se traduit par une transformation majeure de l'organisation et du contenu des études.

\section{INDEX}

Mots-clés : université, formation, professionnalisation 


\section{AUTEURS}

\section{MATTHIEU PICHON}

Doctorant, Université Paris 1, UMR Géographie-Cités

matthieu.pichon@univ-paris1.fr

\section{CAROLINE LEININGER-FRÉZAL}

Maître de conférences, Université Paris-Diderot, EA LDAR

carolinefrezal@wanadoo.fr

\section{NICOLAS DOUAY}

Maître de conférences, Université Paris-Diderot, UMR Géographie-Cités nicolas.douay@gmail.com 